\title{
Rubidium and zirconium abundances in massive Galactic asymptotic giant branch stars revisited
}

\author{
V. Pérez-Mesa ${ }^{1,2}$, O. Zamora ${ }^{1,2}$, D. A. García-Hernández ${ }^{1,2}$, B. Plez ${ }^{3}$, A. Manchado ${ }^{1,2,4}$, \\ A. I. Karakas ${ }^{5}$, and M. Lugaro 5,6 \\ ${ }^{1}$ Instituto de Astrofísica de Canarias (IAC), 38205 La Laguna, Tenerife, Spain \\ 2 Departamento de Astrofísica, Universidad de La Laguna (ULL), 38206 La Laguna, Tenerife, Spain \\ 3 Laboratoire Univers et Particules de Montpellier, Université de Montpellier 2, CNRS, 34095 Montpellier, France \\ ${ }^{4}$ Consejo Superior de Investigaciones Científicas (CSIC), 28006 Madrid, Spain \\ 5 Monash Centre for Astrophysics, School of Physics and Astronomy, Monash University, VIC3800, Australia \\ ${ }^{6}$ Konkoly Observatory, Research Centre for Astronomy and Earth Sciences, Hungarian Academy of Sciences, 1121 Budapest,
} e-mail: vperezme@iac.es Hungary

Received 25 May 2017 / Accepted 7 June 2017

\begin{abstract}
Context. Luminous Galactic OH/IR stars have been identified as massive ( $>4-5 M_{\odot}$ ) asymptotic giant branch (AGB) stars experiencing hot bottom burning and $\mathrm{Li}$ production. Their $\mathrm{Rb}$ abundances and $[\mathrm{Rb} / \mathrm{Zr}]$ ratios, as derived from classical hydrostatic model atmospheres, are significantly higher than predictions from AGB nucleosynthesis models, posing a problem for our understanding of AGB evolution and nucleosynthesis.

Aims. We report new $\mathrm{Rb}$ and $\mathrm{Zr}$ abundances in the full sample (21) of massive Galactic AGB stars, previously studied with hydrostatic models, by using more realistic extended model atmospheres.

Methods. For this, we use a modified version of the spectral synthesis code Turbospectrum and consider the presence of a circumstellar envelope and radial wind in the modelling of the optical spectra of these massive AGB stars. The $\mathrm{Rb}$ and $\mathrm{Zr}$ abundances are determined from the $7800 \AA \mathrm{Rb}$ I resonant line and the $6474 \AA \mathrm{ZrO}$ bandhead, respectively, and we explore the sensitivity of the derived abundances to variations of the stellar $\left(T_{\text {eff }}\right)$ and wind $\left(\dot{M}, \beta\right.$ and $\left.v_{\text {exp }}\right)$ parameters in the pseudo-dynamical models. The Rb and $\mathrm{Zr}$ abundances derived from the best spectral fits are compared with the most recent AGB nucleosynthesis theoretical predictions.

Results. The $\mathrm{Rb}$ abundances derived with the pseudo-dynamical models are much lower (in the most extreme stars even by $\sim 1-2$ dex) than those derived with the hydrostatic models, while the $\mathrm{Zr}$ abundances are similar. The $\mathrm{Rb}$ I line profile and $\mathrm{Rb}$ abundance are very sensitive to the wind mass-loss rate $\dot{M}$ (especially for $\dot{M} \geq 10^{-8} M_{\odot} \mathrm{yr}^{-1}$ ) but much less sensitive to variations of the wind velocity-law $\left(\beta\right.$ parameter) and the expansion velocity $v_{\exp }(\mathrm{OH})$.

Conclusions. We confirm the earlier preliminary results based on a smaller sample of massive O-rich AGB stars, suggesting that the use of extended atmosphere models can solve the discrepancy between the AGB nucleosynthesis theoretical models and the observations of Galactic massive AGB stars. The Rb abundances, however, are still strongly dependent on the wind mass-loss $\dot{M}$, which, unfortunately, is unknown in these AGB stars. Accurate mass-loss rates $\dot{M}$ (e.g. from rotationally excited lines of the CO isotopologues in the radio domain) in these massive Galactic AGB stars are needed in order to break the model's degeneracy and obtain reliable (non-model-dependent) $\mathrm{Rb}$ abundances in these stars.
\end{abstract}

Key words. stars: AGB and post-AGB - stars: atmospheres - stars: abundances - stars: evolution - stars: late-type nuclear reactions, nucleosynthesis, abundances

\section{Introduction}

The asymptotic giant brach (AGB; Herwig 2005; Karakas \& Lattanzio 2014) is occupied by low- and intermediate-mass $\left(0.8 \leq M \leq 8 M_{\odot}\right)$ stars in the last nuclear-burning phase. At the end of the AGB phase, these stars develop thermal pulses (TP) and suffer extreme mass loss. AGB stars are thus one of the main contributors to the enrichment of the interstellar medium (ISM) of light elements (e.g. Li, C, N, F) and heavy (slow neutron capture, $s$-process) elements and thus to the chemical evolution of galaxies (Busso et al. 1999). AGB stars are also one of the most prominent sources of dust in galaxies and the site of origin of the vast majority of meteoritic stardust grains (e.g. Hoppe \& Ott 1997; Nittler et al. 1997; Lugaro et al. 2017). In low-mass AGB stars $\left(M<4 M_{\odot}\right){ }^{12} \mathrm{C}$ is produced during the TP-AGB phase, and carried to the stellar surface via the third dredge-up (TDU) that can occur after each TP, transforming originally $\mathrm{O}$-rich stars into C-rich stars $(\mathrm{C} / \mathrm{O}>1)$ (e.g. Herwig 2005; Karakas \& Lattanzio 2007; Lugaro \& Chieffi 2011). However, the more massive AGB stars $\left(M>4-5 M_{\odot}\right)$ are O-rich $(\mathrm{C} / \mathrm{O}<1)$ because the socalled hot bottom burning (HBB) process is activated. Through the $\mathrm{CN}$ cycle via proton captures at the base of the convective envelope, $\mathrm{HBB}$ converts ${ }^{12} \mathrm{C}$ into ${ }^{13} \mathrm{C}$ and ${ }^{14} \mathrm{~N}$, thus preventing the formation of a carbon star (Sackmann \& Boothroyd 1992; Mazzitelli et al. 1999).

The $s$-process allows the production of neutron-rich elements heavier than iron ( $s$-elements such as $\mathrm{Sr}, \mathrm{Y}, \mathrm{Zr}, \mathrm{Ba}, \mathrm{La}$, $\mathrm{Nd}, \mathrm{Tc}$, etc.) by the $s$-process. In the low-mass AGB stars (roughly $\left.<4 M_{\odot}\right)$, the ${ }^{13} \mathrm{C}(\alpha, \mathrm{n}){ }^{16} \mathrm{O}$ reaction is the dominant neutron source (e.g. Abia et al. 2001). In the more massive 
AGB stars, instead, neutrons are mainly released by the ${ }^{22} \mathrm{Ne}(\alpha$, $\mathrm{n})^{25} \mathrm{Mg}$ reaction, resulting in a higher neutron density (up to $10^{13} \mathrm{n} / \mathrm{cm}^{3}$ ) and temperature environment than in lowermass AGB stars (García-Hernández et al. 2006). The Rb produced depends on the probability of the ${ }^{85} \mathrm{Kr}$ and ${ }^{86} \mathrm{Rb}$ capturing a neutron before decaying and acting as "branching points" (see van Raai et al. 2012, for more details). The probability of this happening depends on the local neutron density (Beer \& Macklin 1989). The ${ }^{87} \mathrm{Rb} /{ }^{85} \mathrm{Rb}$ isotopic ratio is a direct indicator of the neutron density at the production site but it is not possible to distinguish individual ${ }^{87} \mathrm{Rb}$ and ${ }^{85} \mathrm{Rb}$ from stellar spectra (García-Hernández et al. 2006). However, the relative abundance of $\mathrm{Rb}$ to other nearby $s$-process elements such as $\mathrm{Zr}$ is very sensitive to the neutron density, and so a good discriminant of the stellar mass and the neutron source at the $s$-process site (Lambert et al. 1995; Abia et al. 2001; García-Hernández et al. 2006; van Raai et al. 2012). In other words, [Rb/Zr] $<0$ is observed in low-mass AGB stars where the main neutron source is the ${ }^{13} \mathrm{C}(\alpha, \mathrm{n}){ }^{16} \mathrm{O}$ reaction (Plez et al. 1993; Lambert et al. 1995; Abia et al. 2001), while [Rb/Zr] $>0$ is observed in more massive AGB stars, where the neutrons are mainly released through the ${ }^{22} \mathrm{Ne}(\alpha, \mathrm{n}){ }^{25} \mathrm{Mg}$ reaction (García-Hernández et al. 2006, 2007, 2009).

Chemical abundance analyses using classical MARCS hydrostatic atmospheres (Gustafsson et al. 2008) revealed strong $\mathrm{Rb}$ overabundances $\left(\sim 10^{3}-10^{5}\right.$ times solar) and high $[\mathrm{Rb} / \mathrm{Zr}]$ ratios $(\geqslant 3-4 \mathrm{dex})$ in massive AGB stars (generally very luminous $\mathrm{OH} / \mathrm{IR}$ stars) of our own Galaxy and the Magellanic Clouds (MC; García-Hernández et al. 2006, 2007, 2009). This observationally confirmed for the first time that the ${ }^{22} \mathrm{Ne}$ neutron source dominates the production of $s$-process elements in these stars. However, the extremely high $\mathrm{Rb}$ abundances and $[\mathrm{Rb} / \mathrm{Zr}]$ ratios observed in most the massive stars (and especially in the lower-metallicity MC AGB stars) have posed a "Rb problem"; such extreme $[\mathrm{Rb} / \mathrm{Fe}]$ and $[\mathrm{Rb} / \mathrm{Zr}]$ values are not predicted by the $s$-process AGB models, (van Raai et al. 2012; Karakas et al. 2012), suggesting fundamental problems in our present understanding of AGB nucleosynthesis and/or of the complex extended dynamical atmospheres of these stars (García-Hernández et al. 2009).

Zamora et al. (2014) constructed new pseudo-dynamical MARCS model atmospheres by considering the presence of a gaseous circumstellar envelope with a radial wind and applied them to a small sample of five O-rich AGB stars with different expansion velocities and metallicities. The $\mathrm{Rb}$ abundances and $[\mathrm{Rb} / \mathrm{Zr}]$ ratios obtained were much lower than those obtained with classical hydrostatic models; in better agreement with the AGB nucleosynthesis theoretical predictions. In this paper, we use the Zamora et al. (2014) pseudo-dynamical model atmospheres to obtain the abundances of $\mathrm{Rb}$ and $\mathrm{Zr}$ in the full sample of massive Galactic AGB stars previously analysed with hydrostatic models (García-Hernández et al. 2006, 2007). These Rb and $\mathrm{Zr}$ abundances are then compared with the more recent AGB nucleosynthesis theoretical predictions available in the literature.

\section{Sample and observational data}

Our sample is composed of 21 massive Galactic AGB stars (most of them very luminous OH/IR stars) previously analysed by García-Hernández et al. (2006, 2007); we use their high-resolution $(R \sim 40000-50000)$ optical echelle spectra (we refer to García-Hernández et al. 2006, 2007, for further observational details $)^{1}$. The signal-to-noise ratios $(\mathrm{S} / \mathrm{N})$ achieved in the reduced spectra vary greatly from the blue to the red (typically $\sim 10-20$ at $6000 \AA$ and $>100$ at $8000 \AA$ ). The $\mathrm{Rb}$ and $\mathrm{Zr}$ abundances were determined from the resonant $7800 \AA \mathrm{Rb}$ I line and the $6474 \AA \mathrm{ZrO}$ bandhead, respectively, by using classical MARCS hydrostatic model atmospheres (García-Hernández et al. 2006, 2007). The Rb abundances and $[\mathrm{Rb} / \mathrm{Zr}]$ ratios obtained from this chemical analysis are mostly in the range $[\mathrm{Rb} / \mathrm{Fe}] \sim 0.6-2.6 \mathrm{dex}$ and $[\mathrm{Rb} / \mathrm{Zr}] \sim$ $0.1-2.1$ dex. The atmospheric parameters and $\mathrm{Rb}$ abundances derived with the hydrostatic models as well as other useful observational information like the $\mathrm{OH}$ expansion velocity, variability period, and the presence of $\mathrm{Li}$ are listed in Table 1.

\section{Chemical abundance analysis using pseudo-dynamical models}

\subsection{Modified version of the Turbospectrum spectral synthesis code}

We used the v12.2 version of the spectral synthesis code Turbospectrum (Alvarez \& Plez 1998; Plez 2012), which considers the presence of a circumstellar gas envelope and a radial wind, as modified by Zamora et al. (2014). The main modifications are the following: (i) The Doppler effect due to the extended atmosphere and velocity field is introduced in the routines that compute the line intensities at the stellar surface; (ii) the source function of the radiative transfer is assumed to be the same as computed in the static case (Gustafsson et al. 2008). The validity of this approximation was tested by comparing with Monte Carlo simulations (see Zamora et al. 2014); (iii) The scattering term of the source function $\left(\propto \sigma_{\lambda} J_{\lambda}\right)$ is not shifted to save computing time and is only incorporated for the continuum. This scattering term is computed as in the static case using the Feautrier method (Nordlund 1984; Gustafsson et al. 2008); and (iv) the velocity field is taken into account through a shift of the absorption coefficient $\kappa_{\lambda}$; the source function is built using the static $\sigma_{\lambda} J_{\lambda}$ and the shifted $\kappa_{\lambda} B_{\lambda}$. The emerging intensity is then computed in the observer frame by a direct quadrature of the source function.

\subsection{Extended atmosphere models}

For the analysis of each star in our sample, we adopted the atmosphere parameters from García-Hernández et al. (2006, 2007) and the solar reference abundances by Grevesse et al. (2007). We constructed our pseudo-dynamical models from the original MARCS hydrostatic atmosphere model structure. We expanded the atmosphere radius by a wind out to $\sim 5$ stellar radii and a radial velocity field (Zamora et al. 2014). In the MARCS hydrostatic model, the $R_{*}$ is the radius corresponding to $r\left(\tau_{\text {Ross }}=1\right)$, where $r$ is the distance from the centre of the star and $\tau_{\text {Ross }}$ is the Rosseland optical depth. We computed the stellar wind following the mass conservation (Eq. (1)), radiative thermal equilibrium

\footnotetext{
1 The high-resolution spectra were obtained using the Utrecht Echelle Spectrograph (UES) at the $4.2 \mathrm{~m}$ William Herschel Telescope (La Palma, Spain) and the CAsegrain Echelle SPECtrograph (CASPEC) of the ESO $3.6 \mathrm{~m}$ telescope (La Silla, Chile) during several observing periods in 1996-97 (see García-Hernández et al. 2007).
} 
V. Pérez-Mesa et al.: $\mathrm{Rb}$ and $\mathrm{Zr}$ abundances in massive Galactic AGB stars revisited

Table 1. Atmosphere parameters and $\mathrm{Rb}$ abundances (as derived using hydrostatic models) and other selected observational information.

\begin{tabular}{cccccccc}
\hline \hline IRAS name & $T_{\text {eff }}(\mathrm{K})$ & $\log g$ & $v_{\text {exp }}(\mathrm{OH})\left(\mathrm{km} \mathrm{s}^{-1}\right)$ & Period (days) & Lithium & {$[\mathrm{Rb} / \mathrm{Fe}]_{\text {static }}$} & $S / N$ at $7800 \AA$ \\
\hline $01085+3022$ & $3000^{*}$ & -0.5 & 13 & 560 & yes & 2.0 & 49 \\
$04404-7427$ & 3000 & -0.5 & 8 & 534 & $\ldots$ & 1.3 & 68 \\
$05027-2158$ & 2800 & -0.5 & 8 & 368 & yes & 0.4 & 418 \\
$05098-6422$ & 3000 & -0.5 & 6 & 394 & no & 0.1 & 309 \\
$05151+6312$ & 3000 & -0.5 & 15 & $\ldots$ & no & 2.1 & 161 \\
$06300+6058$ & 3000 & -0.5 & 12 & 440 & yes & 1.6 & 127 \\
$07222-2005$ & 3000 & -0.5 & 8 & 1200 & $\ldots$ & 0.6 & 30 \\
$09194-4518$ & 3000 & -0.5 & 11 & $\ldots$ & $\ldots$ & 1.1 & 25 \\
$10261-5055$ & 3000 & -0.5 & 4 & 317 & no & $<-1.0$ & 595 \\
$14266-4211$ & 2900 & -0.5 & 9 & 389 & no & 0.9 & 106 \\
$15193+3132$ & 2800 & -0.5 & 3 & 360 & no & -0.3 & 266 \\
$15576-1212$ & 3000 & -0.5 & 10 & 415 & yes & 1.5 & 91 \\
$16030-5156$ & 3000 & -0.5 & $7-14$ & 579 & yes & 1.3 & 86 \\
$16037+4218$ & 2900 & -0.5 & 4 & 360 & no & 0.6 & 115 \\
$17034-1024$ & 3300 & -0.5 & $3-9$ & 346 & no & 0.2 & 189 \\
$18429-1721$ & 3000 & -0.5 & 7 & 481 & yes & 1.2 & 98 \\
$19059-2219$ & 3000 & -0.5 & 13 & 510 & $\ldots$ & 2.3 & 32 \\
$19426+4342$ & 3000 & -0.5 & 9 & $\ldots$ & $\ldots$ & 1.0 & 19 \\
$20052+0554$ & $3000^{*}$ & -0.5 & 16 & 450 & yes & 1.5 & 47 \\
$20077-0625$ & 3000 & -0.5 & 12 & 680 & $\ldots$ & 1.3 & 19 \\
$20343-3020$ & 3000 & -0.5 & 8 & 349 & no & 0.9 & 76 \\
\hline
\end{tabular}

Notes. The stellar parameters, $\mathrm{Rb}$ abundances, $\mathrm{OH}$ expansion velocities, variability periods and presence of Li are collected from García-Hernández et al. (2006; 2007, and references therein). The asterisks indicate that the best fitting $T_{\text {eff }}$ in the $\mathrm{ZrO} 6474 \AA$ spectral region is warmer (3300 K) than that around the Rb I 7800 Å line (García-Hernández et al. 2006, 2007). Two stars (IRAS 16030-5156 and IRAS 17034-1024) only display the blue-shifted $1612 \mathrm{MHz} \mathrm{OH}$ maser peak and we list the range of $\mathrm{OH}$ expansion velocities shown by other stars with similar variability periods (García-Hernández et al. 2007).

(Eq. (2)) and a classical $\beta$-velocity law (Eq. (3)),

$\rho(r)=\frac{\dot{M}}{4 \pi r^{2} v(r)}$,

$r T^{2}=$ constant $=r_{\text {out }} T_{\text {out }}^{2}$,

$v(r)=v_{0}+\left(v_{\infty}-v_{0}\right)\left(1-\frac{R_{*}}{r}\right)^{\beta}$,

where $\rho(r)$ is the density of the envelope radius $r, \dot{M}$ is the massloss rate, and $v(r)$ is the velocity of the envelope, which is calculated by means of Eq. (3). In Eq. (3), $v_{0}$ is a reference velocity for the beginning of the wind and $\beta$ is an arbitrary free parameter. We take $v_{0}=v\left(R_{*}\right)$ for the onset of the wind and the extension of the envelope begins from the outer radius of the hydrostatic model. Using Eq. (2) the envelope is extended, layer by layer, out to the distance $r_{\max }$, which corresponds to the maximum radius in our calculations, with $T_{\min }=1000 \mathrm{~K}$. Turbospectrum cannot compute lower temperatures due to numerical reasons (Zamora et al. 2014).

\subsection{Resulting grids of synthetic spectra}

The synthetic spectra are generated with the modified version of Turbospectrum by using the extended pseudo-dynamical model atmospheres as input. We constructed a mini-grid of synthetic spectra for each sample star by adopting the atmospheric parameters (e.g. effective temperature, macroturbulence ${ }^{2}$ ) from García-Hernández et al. (2006, 2007). Basically, the stellar mass, gravity $\log g$, microturbulent velocity $\xi$, metallicity $[\mathrm{Fe} / \mathrm{H}]$, and $\mathrm{C} / \mathrm{O}$ ratio are fixed to $2 M_{\odot},-0.5 \mathrm{dex}, 3 \mathrm{~km} \mathrm{~s}^{-1}, 0.0$, and 0.5 dex, respectively (we refer to García-Hernández et al. 2007 , for more details). On the other hand, for the mass-loss rate $\dot{M}$ and the exponent $\beta$, we use values in the range $\dot{M} \sim 10^{-9}$ $10^{-6} M_{\odot} \mathrm{yr}^{-1}$ in steps of $0.5 \times 10^{-1} M_{\odot} \mathrm{yr}^{-1}$ and the range $\beta \sim$ $0.2-1.6$ in steps of 0.2 . We have not considered the case where $\beta=0.0$ because the expansion velocity would be constant at any $r$. We assume the $\mathrm{OH}$ expansion velocity $\left(v_{\exp }(\mathrm{OH})\right.$; see Table 1$)$ as the terminal velocity because the $\mathrm{OH}$ maser emission is found at very large distances from the central star (e.g. Decin et al. 2010). Figure 1 shows examples of the $\beta$-velocity laws used in our pseudo-dynamical models based on the MARCS hydrostatic models. Finally, for the $\mathrm{Rb}$ and $\mathrm{Zr}$ abundances, we used $[\mathrm{Rb} / \mathrm{Fe}] \sim-2.6$ to +3.0 dex, and $[\mathrm{Zr} / \mathrm{Fe}] \sim-1.0$ to +1.0 in steps of 0.1 and 0.25 dex, respectively.

The resulting mini-grid ( 4400 models) is compared to the observed spectrum in order to find the synthetic spectrum that best fits the $7800 \AA \mathrm{Rb}$ I line and the $6474 \AA \mathrm{ZrO}$ bandhead

\footnotetext{
2 The synthetic spectra are convolved with a Gaussian profile (with a certain FWHM typically between 250 and $400 \mathrm{~m} \AA$ ) to account for macroturbulence as well as instrumental profile effects.
} 


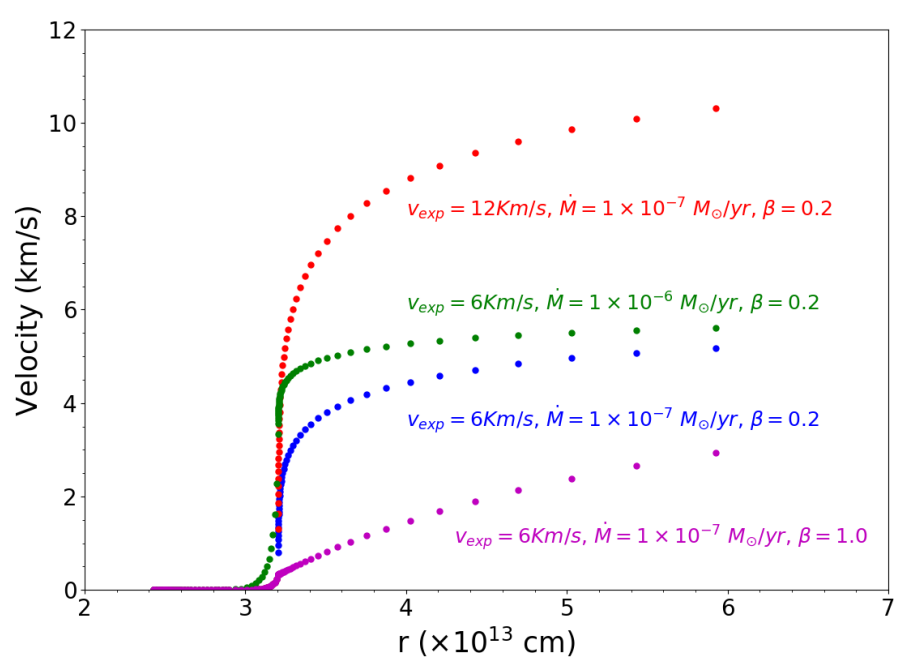

Fig. 1. Velocity vs. distance from the star in four of our AGB wind models. These velocity laws present different expansion velocities $v_{\exp }(\mathrm{OH})$, mass-loss rates $\dot{M}$, and $\beta$ exponents. The effective temperature $T_{\text {eff }}=$ $3000 \mathrm{~K}$, gravity $\log g=-0.5$ and the solar chemical composition are the same in all models.

profiles and their adjacent pseudocontinua. In order to obtain the best fits, we made use of a procedure based on the comparison between synthetic and observed spectra, while in Zamora et al. (2014) the observed spectra were fitted by eye. The method is a modified version of the standard $\chi^{2}$ test,

$\chi^{2 *}=\chi^{2} \times w=\left(\sum_{i=1}^{N} \frac{\left[\operatorname{Yobs}_{i}-\operatorname{Ysynth}_{i}\left(x_{1} \ldots x_{M}\right)\right]^{2}}{\operatorname{Yobs}_{i}}\right) \times w$,

where $Y o b s_{i}$ and $Y s y n t h_{i}$ are the observed and synthetic data points, respectively, with $N$ the number of data points, and $M$ the number of free parameters. On the other hand, $w$ is a vector that gives a stronger weight to the detailed spectral profiles of the $\mathrm{Rb}$ I line and the $\mathrm{ZrO}$ bandhead. This way, the lowest value of $\chi^{2 *}$ gives us the best fitting synthetic spectrum from the minigrid for each sample star.

The use of the $\chi^{2 *}$ test to find the best fits to the observed spectra reveals the presence of important degeneracies in the resulting grids of pseudo-dynamical synthetic spectra; that is, very similar synthetic spectra are obtained from different sets of wind parameters (more details below). Moreover, in a some cases (IRAS 04404-7427, IRAS 05027-2158, IRAS 05098-6422, IRAS 06300+6058, IRAS 10261-5055, IRAS 18429-1721, IRAS 19059-2219 and IRAS 20343-3020) the use of the $\chi^{2 *}$ test is not enough for obtaining the synthetic spectrum that best reproduces the observed one and the best fits have to be found by eye. Unfortunately, the wind model parameters $\dot{M}$ and $\beta$ are generally not known for stars in our sample (see below), complicating the abundance analysis. Thus, here we study the sensitivity of the synthetic spectra and the abundance results to variations of the stellar and wind parameters.

\subsection{Sensitivity of the synthetic spectra to variations of the model parameters}

Here, we analyse how the variations in stellar $\left(T_{\text {eff }}\right)$ and wind $(\dot{M}$, $\beta$ and $\left.v_{\exp }(\mathrm{OH})\right)$ parameters influence the output synthetic spectra. Figures 2 and 3 show examples of synthetic spectra for different stellar and wind parameters in the spectral regions around the $7800 \AA \mathrm{Rb}$ I line and $6474 \AA \mathrm{ZrO}$ bandhead, respectively. We note that the fraction of the absortion at $7800 \AA$ due to other species (e.g. TiO) is typically around $20 \%$.

The Rb I line profile is very sensitive to the wind mass-loss rate $\dot{M}$ (especially for $\dot{M} \geq 10^{-8} M_{\odot} \mathrm{yr}^{-1}$ ); the $\mathrm{Rb}$ I line is significantly deeper and blue-shifted with increasing $\dot{M}$ (Fig. 2, top-left panel). However, the $\mathrm{Rb}$ I line profile is much less sensitive to changes of the wind velocity-law ( $\beta$ parameter); being only slightly deeper with increasing $\beta$ (Fig. 2, bottom-left panel). In addition, for $\beta$ values higher than $\sim 1.2$ (shallower velocity profiles), the $\mathrm{Rb}$ I line profile is not sensitive to variations of the expansion velocity $v_{\exp }(\mathrm{OH})$ because the velocity profiles are very similar in our extended model atmosphere (up to $\sim 10^{14} \mathrm{~cm}$; see Fig. 1). Variations in the expansion velocity $v_{\text {exp }}(\mathrm{OH})$ mainly affect the blue-shift of the Rb I line and, in addition, for large $v_{\exp }(\mathrm{OH})$ values the core of the $\mathrm{Rb}$ I line is less deep (Fig. 2, bottom-right panel). Finally, the $\mathrm{Rb}$ I absorption line is stronger with decreasing effective temperature $T_{\text {eff }}$ (as expected; Fig. 2, top-right panel) but this time the wealth of $\mathrm{TiO}$ molecular lines and the pseudo-continua are also affected. We note that all these effects (variations in the $\mathrm{Rb}$ I profile in terms of depth and blue-shift) are more evident for extreme mass-loss rates $\left(\dot{M} \geq 10^{-7} M_{\odot} \mathrm{yr}^{-1}\right)$ and higher Rb abundances.

On the other hand, the $\mathrm{ZrO}$ bandhead profile is not sensitive to the wind parameters $\dot{M}, \beta$, and $v_{\text {exp }}(\mathrm{OH})$ (see Fig. 3; topleft, bottom-left, and bottom-right panels, respectively). The $\mathrm{ZrO}$ bandhead profile (as well as the adjacent TiO lines and pseudocontinuum) are, again as expected, stronger with decreasing $T_{\text {eff }}$ (Fig. 3, top-right panel). This is because $\mathrm{ZrO}$ is formed deeper than $\mathrm{Rb}$ I in the atmosphere, being much less affected by the circumstellar envelope and radial wind.

\section{Results}

As we have mentioned above, there are important degeneracies in the resulting mini-grids of synthetic spectra for each star. Two synthetic spectra with the same $T_{\text {eff }}, \log g$ and $v_{\exp }(\mathrm{OH})$, but different $\beta, \dot{M}$, and $[\mathrm{Rb} / \mathrm{Fe}]$ abundances could be practically identical in spite of the different wind parameters. This complicates the abundance analysis because the wind model parameters $\dot{M}$ and $\beta$ are generally not known for stars in our sample. In any case, we can use some observational constraints and previous results on a few similar $\mathrm{OH} / \mathrm{IR}$ stars to limit the possible variation range of these wind parameters (in particular for the mass-loss rates $\dot{M}$, see below).

By using multiple rotationally excited lines of both ${ }^{12} \mathrm{CO}$ and ${ }^{13} \mathrm{CO}$, De Beck et al. (2010) provide accurate mass-loss rates $\dot{M}$ for a large sample of Galactic AGB stars. Unfortunately, only one star (IRAS 20077-0625) from our present sample of $\mathrm{Rb}$-rich $\mathrm{OH} / \mathrm{IR}$ massive AGB stars is included in their work and we cannot fit this star with our pseudo-dynamical models (see below). There are seven massive AGB stars of OH/IR type (WX Psc, V669 Cas, NV Aur, OH 26.5+0.6, OH 44.8-2.3, IRC -10529 and $\mathrm{OH} 104.9+2.4)$ previously studied in the optical by García-Hernández et al. (2007). Their variability periods and mass-loss rates range from 552 to 1620 days $^{3}$ and from $1.8 \times 10^{-5} M_{\odot} \mathrm{yr}^{-1}$ to $9.7 \times 10^{-6} M_{\odot} \mathrm{yr}^{-1}$, respectively. Interestingly, all these stars are extremely obscured in the optical, being

\footnotetext{
3 The variability periods of our sample stars are also lower, from $\sim 320$ to 580 days (only two stars display periods in excess of 580 days; Table 1).
} 

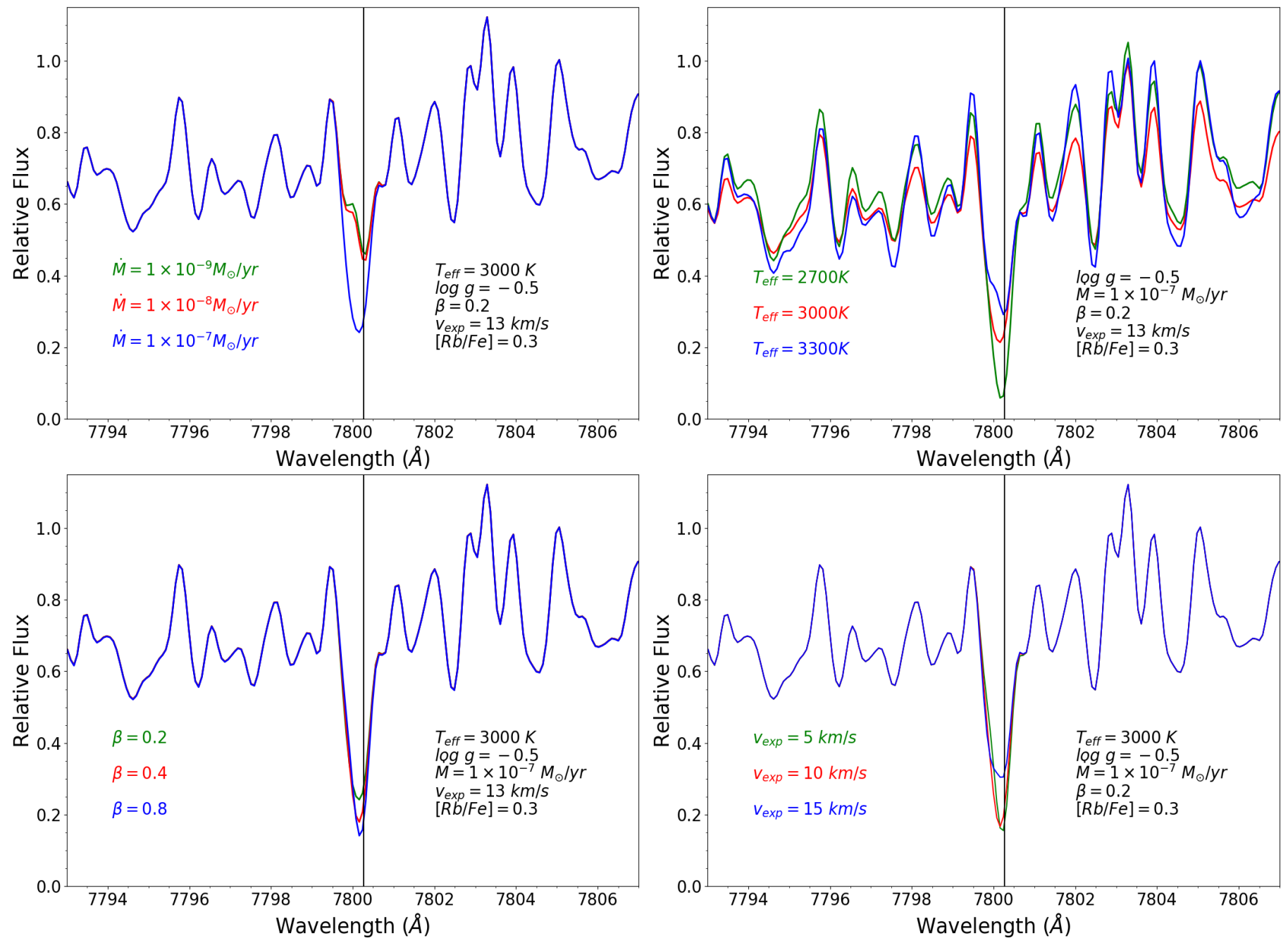

Fig. 2. Illustrative examples of synthetic spectra for different stellar $\left(T_{\text {eff }}\right)$ and wind $\left(\dot{M}, \beta\right.$, and $\left.v_{\text {exp }}(\mathrm{OH})\right)$ parameters in the spectral region around the $7800 \AA \mathrm{Rb}$ I line. The black vertical line indicates the position of the $7800 \AA \mathrm{Rb}$ I line.

too red or without optical counterpart ${ }^{4}$; they likely already have entered the superwind phase. Thus, the $\dot{M}$ values in optically obscured $\mathrm{OH} / \mathrm{IR}$ AGB stars can be taken as upper limits (i.e. $<10^{-6} M_{\odot} \mathrm{yr}^{-1}$ ) for our sample of $\mathrm{OH} / \mathrm{IR}$ massive AGB stars with optical counterparts; that is, with useful spectra around the $7800 \AA \mathrm{Rb}$ I line. Indeed, we generally find that lower mass-loss rates $\left(\sim 10^{-7}-10^{-8} M_{\odot} \mathrm{yr}^{-1}\right)$ give superior fits to the observed $\mathrm{Rb}$ I line profiles. Mass-loss rates of $\sim 10^{-6} M_{\odot} \mathrm{yr}^{-1}$ (or higher) give strong $\mathrm{Rb}$ I absorption lines for solar $\mathrm{Rb}$ abundances (see also Zamora et al. 2014) with the consequence that all stars in our sample of $\mathrm{OH} / \mathrm{IR}$ massive AGB stars would be Rb-poor. By combining the variability periods from Table 1 and the mass-loss rates estimated from the $\mathrm{Rb}$ I line profiles (mainly in the range $\sim 10^{-7}-10^{-8} M_{\odot} \mathrm{yr}^{-1}$; Table 2) into the AGB mass-loss formula by Vassiliadis and Wood (1993, their Eq. (5)), we obtain reasonable current stellar masses in the range $\sim 2.5-6 M_{\odot}$. In Table 2 we show the mass-loss rates obtained from the best spectral fits $\left(\dot{M}_{f i t}\right)$ and the current stellar masses by using the mass-loss expression from Vassiliadis \& Wood (1993).

4 The only exception is WX Psc as already noted by Zamora et al. (2014). This star (with a mass-loss rate of $\sim 1.8 \times 10^{-5} M_{\odot} \mathrm{yr}^{-1}$, De Beck et al. 2010; Justtanont et al. 2013) has an extremely faint optical counterpart. The $\mathrm{S} / \mathrm{N}$ around $7800 \AA$ is too low for an abundance analysis but a strong $\mathrm{Rb}$ I absorption line is clearly detected in its optical spectrum.
The $\beta$ parameter in our models (only up to $\sim 10^{14} \mathrm{~cm}$ from the photosphere; Fig. 1) cannot be directly compared with other estimations of this parameter in the literature (e.g. Decin et al. 2010; Danilovich et al. 2015), which map many outer regions in the circumstellar envelope and that usually obtain relatively high and uncertain values $(0 \leq \beta \leq 5.0)$. However, the effect of the $\beta$ parameter on our synthetic spectra is minor compared to the mass-loss rate $\dot{M}$ and we keep it as a free parameter in our abundance analysis. We note also that the velocity profiles are very similar in our extended model atmosphere for $\beta \geq 1.2$; that is, the $\mathrm{Rb}$ I line profile is not more sensitive to variations of the expansion velocity and the abundance results are very similar for $\beta \geq 1.2$. We generally find better fits with low $\beta$ values (or steeper velocity profiles; see Table 3 ).

As mentioned above, the parameters of the hydrostatic models providing the best fit to the observations and the $\mathrm{Rb}$ abundances derived are shown in Table 1 . The static models use the solar abundances from Grevesse \& Sauval (1998) for computing the Rb abundances (García-Hernández et al. 2006, 2007), while our pseudo-dynamical models use the more recent solar abundances from Grevesse et al. (2007). In Zamora et al. (2014) we compared the Rb abundances from static models using Grevesse \& Sauval (1998) and Grevesse et al. (2007), and the $\mathrm{Rb}$ abundances obtained agree within $\sim 0.2$ dex in most cases.

Figure 4 shows that our pseudo-dynamical atmosphere models reproduce the observed $7800 \AA \mathrm{Rb}$ I line profile much better 

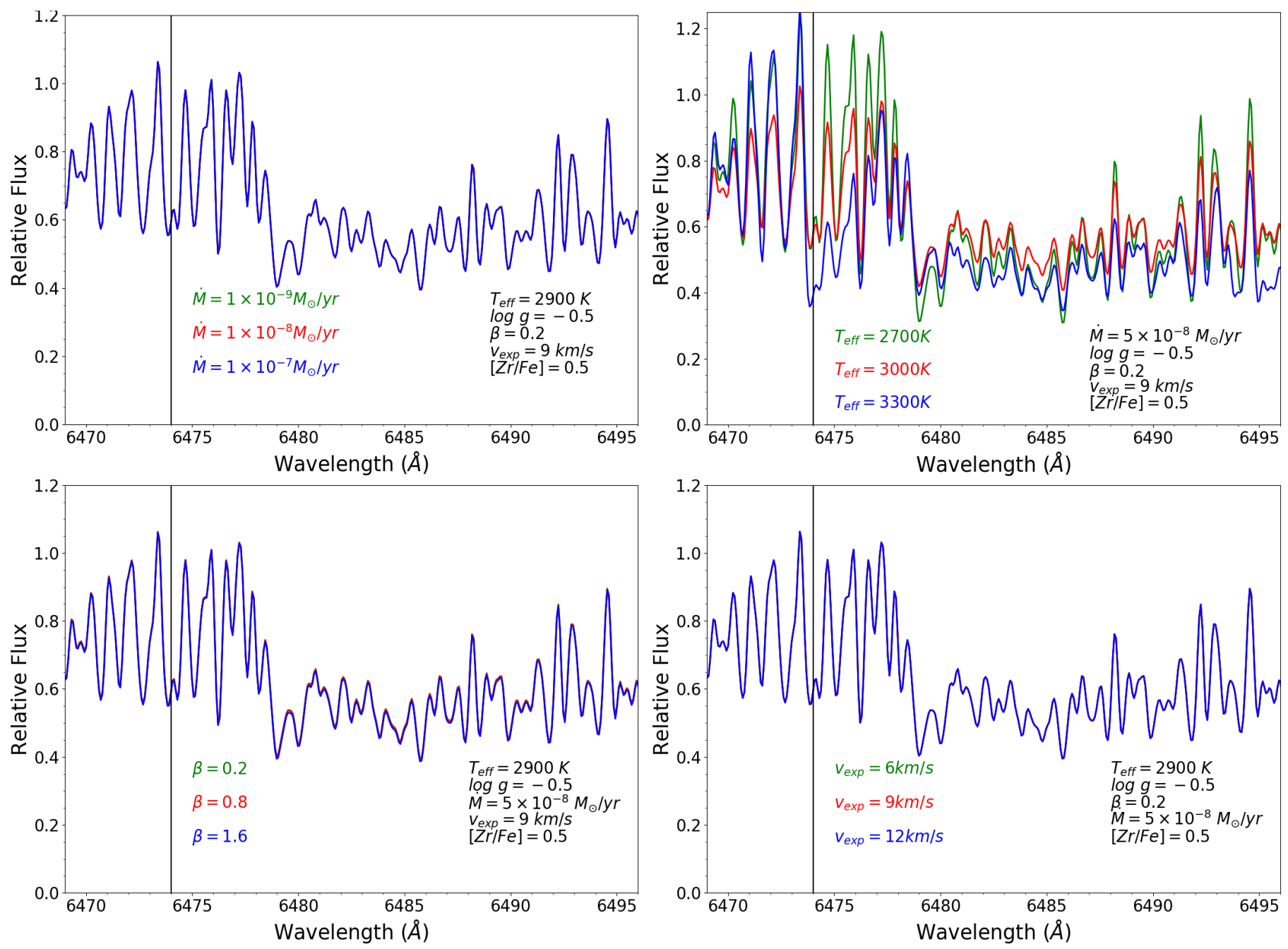

Fig. 3. Illustrative examples of synthetic spectra for different stellar $\left(T_{\text {eff }}\right)$ and wind $\left(\dot{M}, \beta\right.$, and $\left.v_{\text {exp }}(\mathrm{OH})\right)$ parameters in the spectral region around the $6474 \AA \mathrm{ZrO}$ bandhead. The black vertical line indicates the position of the $6474 \AA \mathrm{ArO}$ bandhead.

than the classical hydrostatic models in four sample stars (we refer to Appendix A for the remainder of the sample stars). On the other hand, the $\mathrm{Zr}$ abundances derived from the extended models are similar to those obtained with the hydrostatic models because the $6474 \AA \mathrm{ZrO}$ bandhead is formed deeper in the atmosphere and is less affected by the radial velocity field (Zamora et al. 2014). We could obtain the $\mathrm{Rb}$ and $\mathrm{Zr}$ abundances (or upper limits) for 17 sample stars. The remainder of our sample stars (IRAS 07222-2005, IRAS 09194-4518, IRAS $19426+4342$ and IRAS 20077-0625) seem to display different $\mathrm{Rb}$ I line profiles (e.g. with more than one circumstellar contribution or anomalously broad profiles with red-extended wings; e.g. Fig. 5) that cannot be completely reproduced by our present version of the spectral synthesis code. In the two stars (IRAS 07222-2005 and IRAS 09194-4518) shown in Fig. 5 we cannot fit the two Rb I components (circumstellar and photospheric) at the same time; for example, we could only partially fit the blue-shifted circumstellar component using larger massloss rates $\left(>10^{-6} M_{\odot} \mathrm{yr}^{-1}\right)$. Curiously, these two stars present the largest periods (see Table 1) and they may be the most extreme and evolved stars in our sample, where our extended models do not work so well (e.g. due to even more extended atmospheres). It is not completely clear, however, if the observed profiles are real because these four sample stars have the lowestquality spectra $(S / N<30$ at $7800 \AA$; Table 1$)$.
For the two sample stars with unknown $\mathrm{OH}$ expansion velocity, IRAS 16030-5156 and IRAS 17034-1024, we explore the velocity range displayed by other stars with similar variability periods (see Table 1). Similar fits can be obtained for $v_{\exp }(\mathrm{OH}) \sim 7-12$ and 7-9 $\mathrm{km} \mathrm{s}^{-1}$ (in combination with sligthly different wind parameters) for IRAS 16030-5156 and IRAS 17034-1024, respectively, and we thus adopt average velocities of 10 and $8 \mathrm{~km} \mathrm{~s}^{-1}$, respectively, in the abundance analysis (Table 3).

Table 3 shows the atmospheric and wind parameters as well as the $\mathrm{Rb}$ and $\mathrm{Zr}$ abundances (or upper limits) from the best fits to the observed spectra when the wind parameters $\dot{M}$ and $\beta$ are not fixed. In most cases, the best fit is obtained for both low $\beta(\sim 0.2)$ and $\dot{M}\left(\sim 10^{-9}-10^{-7} M_{\odot} \mathrm{yr}^{-1}\right)$ values. The new $\mathrm{Rb}$ abundances obtained from extended models are lower than those obtained using the hydrostatic models, and the difference is larger for stars with higher hydrostatic $\mathrm{Rb}$ abundances. In addition, this difference is smaller for lower $v_{\exp }(\mathrm{OH})$ and increases with increasing $v_{\exp }(\mathrm{OH})$, as expected. On the other hand, in the case of $\mathrm{Zr}$ we obtain upper limits mostly between 0.0 and +0.25 dex, as derived from the hydrostatic models. Figure 6 displays the hydrostatic and pseudo-dynamical Rb abundances versus the $\mathrm{OH}$ expansion velocity for the wind parameters that provide the best fits (Table 3 ). We plot the $\mathrm{Rb}$ abundances versus the expansion velocity because the $v_{\exp }(\mathrm{OH})$ can 

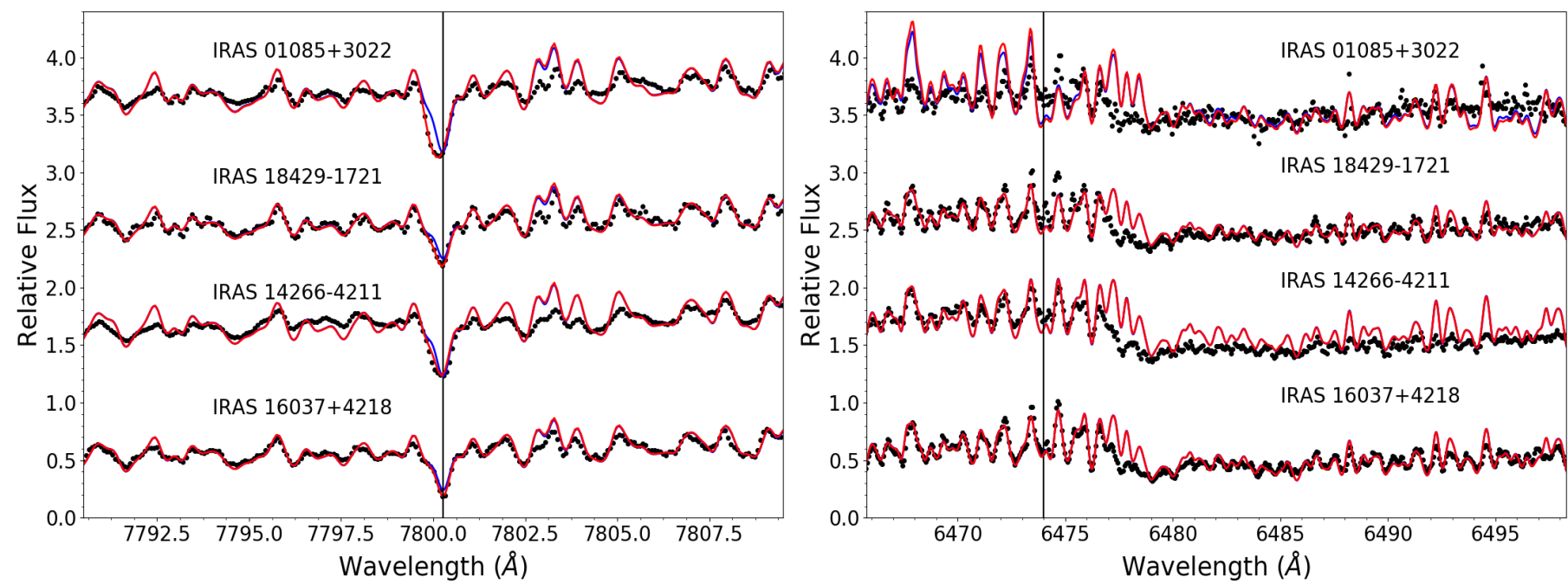

Fig. 4. Rb I $7800 \AA$ (left panel) and ZrO $6474 \AA$ (right panel) spectral regions in massive Galactic AGB stars. The pseudo-dynamical models (red lines) that best fit the observations (black dots) are shown in four sample stars. For comparison, the hydrostatic models are also displayed (blue lines). The location of the $\mathrm{Rb} \mathrm{I}$ line and the $\mathrm{ZrO}$ bandhead are indicated by black vertical lines.
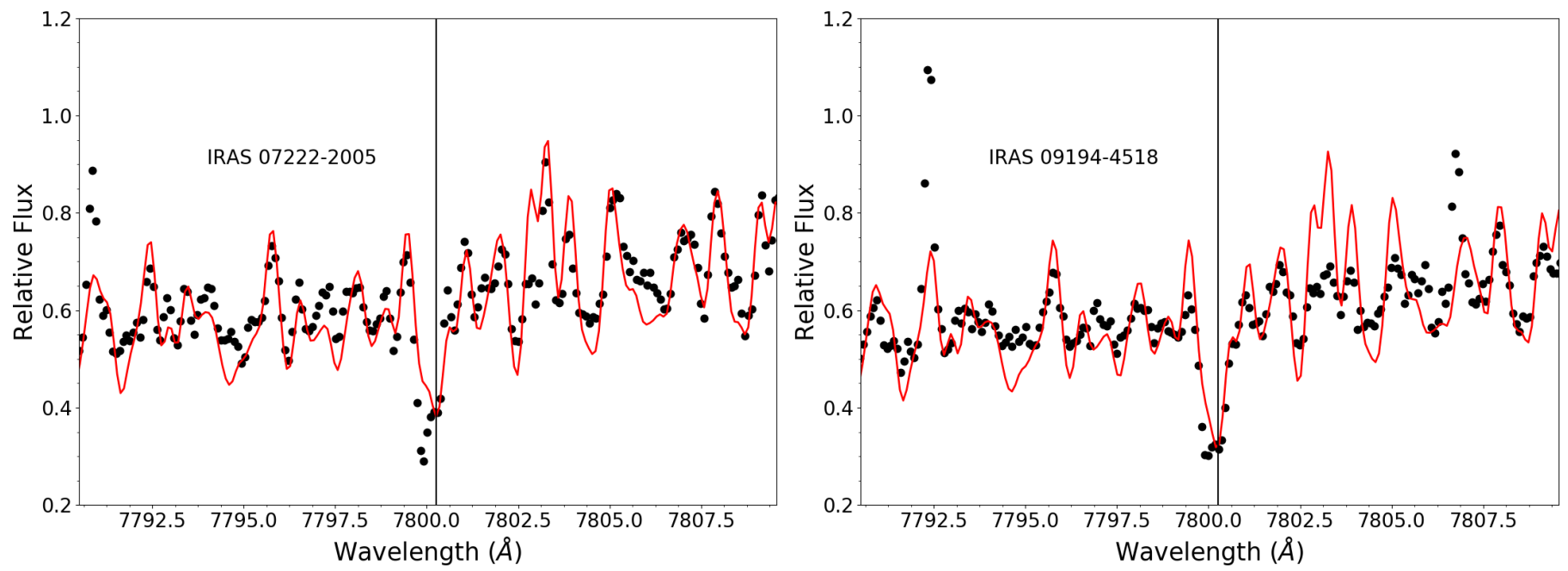

Fig. 5. IRAS 07222-2005 (left panel) and IRAS 09194-4518 (right panel) display two components (circumstellar and photospheric) in the $7800 \AA$ $\mathrm{Rb}$ I line (black line) and cannot be reproduced by our present version of the spectral synthesis code. The red line shows the pseudo-dynamical synthetic spectrum that gives a good fit to the photospheric component only.

be used as a mass indicator independent of the distance in $\mathrm{OH} / \mathrm{IR}$ stars (García-Hernández et al. 2007). In addition, in Fig. 6 we have marked the Li-rich stars (García-Hernández et al. 2007) by squares. About half of the stars with $v_{\exp }(\mathrm{OH})>6 \mathrm{~km} \mathrm{~s}^{-1}$ are Li-rich and most of these stars are also the more $\mathrm{Rb}$-rich ones. We get pseudo-dynamical abundances lower than the hydrostatic ones and a worse correlation between ${ }^{5}$ the $\mathrm{Rb}$ abundances and $v_{\exp }(\mathrm{OH})$; the $\mathrm{Rb}-v_{\exp }(\mathrm{OH})$ relationship is flatter (with a higher degeneration) for the pseudo-dynamical case (see also Sect. 5).

Also, we carried out several tests with different $\beta$ and $\dot{M}$ values in order to check the sensitivity of the derived abundances to variations of the wind parameters. In Table 4 we present the wind parameters and $\mathrm{Rb}$ abundances obtained when fixing $\beta$ to 0.2 and 1.2. Basically, the $\mathrm{Rb}$ abundances are lower in the $\beta=1.2$ case because a higher $\beta$ deepens the Rb I $7800 \AA$ line for the same $\mathrm{Rb}$ abundance (see Fig. 2); in a few cases, however, the $\dot{M}$ of the best fit also changes, further affecting the determination

\footnotetext{
5 The correlation coefficients are $r=0.84$ and 0.54 for the hydrostatic and pseudo-dynamic cases, respectively.
}

of the $\mathrm{Rb}$ abundance. The $\mathrm{Zr}$ abundances (or upper limits) are similar in most cases; the upper limits only change when the $\dot{M}$ is not the same for the $\beta=0.2$ and 1.2 cases. Figure 7 represents the $\mathrm{Rb}$ abundances obtained versus $v_{\exp }(\mathrm{OH})$ for $\beta=0.2$ and $\beta=1.2$. By comparing the $\mathrm{Rb}$ abundances from Figs. 6 and 7 , it is clear that the $\mathrm{Rb}$ abundances are slightly lower in the $\beta=1.2$ case. Moreover, the correlation between the pseudodynamical $\mathrm{Rb}$ abundances and $v_{\exp }(\mathrm{OH})$ for different $\beta$ values is worse (e.g. flatter) than the hydrostatic case. In addition, the dispersion seems to be larger for the $\beta=1.2$ case.

On the other hand, Fig. 8 displays the Rb results when $\dot{M}$ is fixed to $10^{-8}, 10^{-7}$ and $10^{-6} M_{\odot} \mathrm{yr}^{-1}$. This could be equivalent to considering that our AGB sample stars are at a similar evolutionary stage in terms of mass loss; of course we have a strong degeneracy between the progenitor masses and mass loss/evolutionary stage. In the particular case of the Lirich AGB stars, statistical arguments suggest that these stars should have a narrow initial mass range (Di Criscienzo et al. 2016); 4-5 or 5-6 $M_{\odot}$ according to the most recent ATON (Di Criscienzo et al. 2016) or Monash (Karakas \& Lugaro 2016) 
Table 2. Mass-loss rates estimated from the best spectral fits and current stellar masses obtained by using the Vassiliadis \& Wood (1993) massloss formula (their Eq. (5)).

\begin{tabular}{cccc}
\hline \hline IRAS name & $\begin{array}{c}\text { Period } \\
(\text { day })\end{array}$ & $\begin{array}{c}\dot{M} \\
\left(M_{\odot} \mathrm{yr}^{-1}\right)\end{array}$ & $\begin{array}{c}M_{\text {current }} \\
\left(M_{\odot}\right)\end{array}$ \\
\hline $01085+3022$ & 560 & $1.0 \times 10^{-7}$ & 4.6 \\
$04404-7427$ & 534 & $1.0 \times 10^{-7}$ & 4.3 \\
$05027-2158$ & 368 & $1.0 \times 10^{-7}$ & 2.7 \\
$05098-6422$ & 394 & $5.0 \times 10^{-7}$ & 2.4 \\
$05151+6312$ & $\ldots$ & $1.0 \times 10^{-8}$ & $\ldots$ \\
$06300+6058$ & 440 & $1.0 \times 10^{-7}$ & 3.4 \\
$07222-2005$ & 1200 & $\ldots$ & $\ldots$ \\
$09194-4518$ & $\ldots$ & $\ldots$ & $\ldots$ \\
$10261-5055$ & 317 & $1.0 \times 10^{-9}$ & 3.8 \\
$14266-4211$ & 389 & $5.0 \times 10^{-8}$ & 3.1 \\
$15193+3132$ & 360 & $1.0 \times 10^{-9}$ & 4.2 \\
$15576-1212$ & 415 & $1.0 \times 10^{-8}$ & 3.9 \\
$16030-5156$ & 579 & $1.0 \times 10^{-8}$ & 5.6 \\
$16037-1024$ & 360 & $1.0 \times 10^{-7}$ & 2.6 \\
$17034-1024$ & 346 & $1.0 \times 10^{-8}$ & 3.2 \\
$18429-1721$ & 481 & $1.0 \times 10^{-8}$ & 4.6 \\
$19059-2219$ & 510 & $5.0 \times 10^{-8}$ & 4.3 \\
$19426+4342$ & $\ldots$ & $\ldots$ & $\ldots$ \\
$20052+0554$ & 450 & $5.0 \times 10^{-7}$ & 2.9 \\
$20077-0625$ & 680 & $\ldots$ & $\ldots$ \\
$20343-3020$ & 349 & $1.0 \times 10^{-9}$ & 4.1 \\
\hline & & &
\end{tabular}

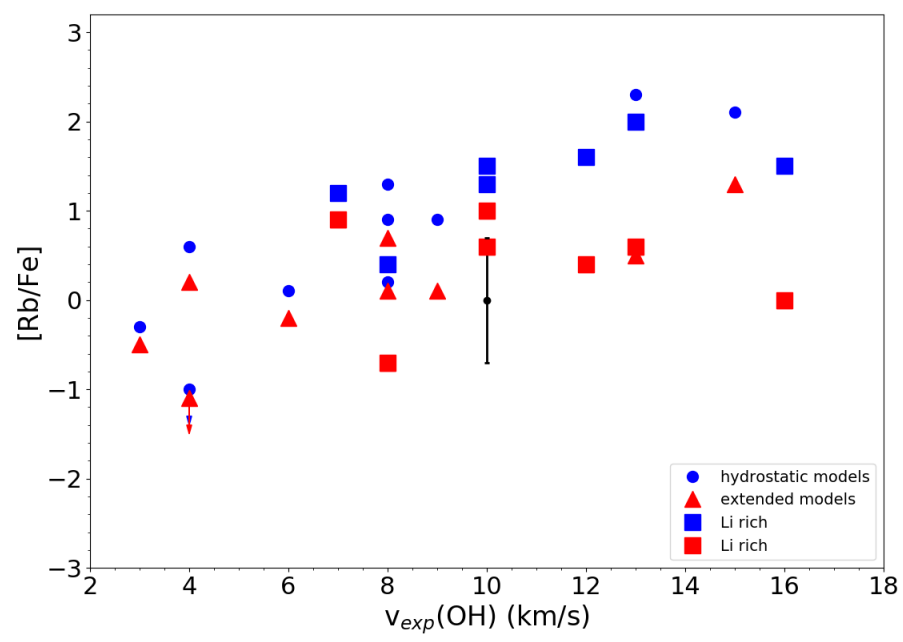

Fig. 6. Rb abundances derived both with hydrostatic (blue dots) and pseudo-dynamical model atmospheres (red triangles) with best-fit parameters plotted against the $\mathrm{OH}$ expansion velocity. The $\mathrm{Li}$-rich stars are indicated by squares. A typical error bar of $\pm 0.7 \mathrm{dex}$ is also displayed.

AGB nucleosynthesis models, respectively. The current stellar masses from Table 2 show, however, that there is a complicated interplay (degeneracy) between Li enhancement, progenitor mass, and mass-loss rate and that the progenitor mass range of these stars may actually be broader; for example,

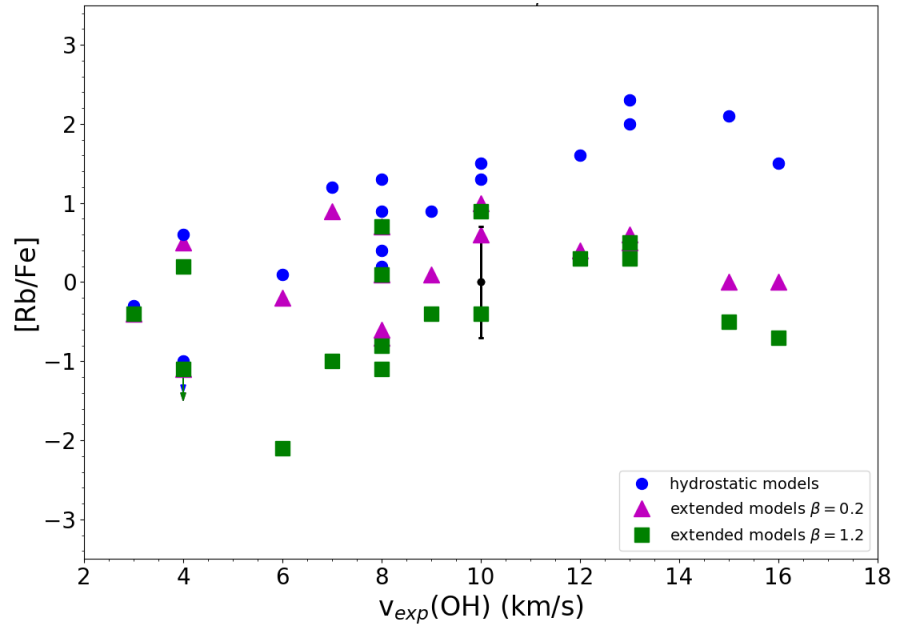

Fig. 7. $\mathrm{Rb}$ abundances derived both with hydrostatic (blue dots) and extended model atmospheres with $\beta=0.2$ (magenta triangles) and $\beta=$ 1.2 (green squares) plotted against the $\mathrm{OH}$ expansion velocity.

their current stellar mass and $\mathrm{Li}$ abundance ranges are $\sim 2.7-$ $5.6 M_{\odot}$ and $\sim \log \varepsilon(\mathrm{Li}) \sim 0.7-2.6$ dex. Figure 8 shows that the $\mathrm{Rb}$ abundances decrease with increasing $\dot{M}$ and the dispersion of the $\mathrm{Rb}$ abundances is much lower when fixing $\dot{M}$. The slopes (and correlation coefficients) of the $\mathrm{Rb}-v_{\exp }(\mathrm{OH})$ correlations are more similar to the ones obtained with hydrostatic models. The $\mathrm{Rb}$ abundances from extended models approach the hydrostatic ones with decreasing $\dot{M}$ (both sets of Rb abundances are identical for $\dot{M} \leq 10^{-9} M_{\odot} \mathrm{yr}^{-1}$ ) because the atmosphere is less extended with decreasing $\dot{M}$, as expected.

Finally, we fixed $\dot{M}$ and $\beta$, which could be equivalent to considering that our AGB sample stars have the same massloss stage and velocity profile. Figure 9 displays the pseudodynamical $\mathrm{Rb}$ abundances versus $v_{\exp }(\mathrm{OH})$ for $\beta=0.2$ and $\dot{M}$ values of $10^{-8}, 10^{-7}$ and $10^{-6} M_{\odot} \mathrm{yr}^{-1}$. The $\mathrm{Rb}$ results, when fixing both $\dot{M}$ and $\beta$, are very similar (with a slightly tighter correlation with $v_{\exp }(\mathrm{OH})$ ) to those obtained when only fixing $\dot{M}$ (see Fig. 8) because $\beta=0.2$ is the most common value obtained from the best spectral fits (all wind parameters free); an exception is the AGB star IRAS $15193+3132$ (with the lowest $v_{\text {exp }}(\mathrm{OH})$ and high $\beta$ ) for which only an upper limit to the $\mathrm{Rb}$ abundance $([\mathrm{Rb} / \mathrm{Fe}] \leq 0.7)$ could be obtained because the pseudodynamical model does not converge for such an unusual combination of wind parameters $\left(\dot{M}=10^{-6} M_{\odot} \mathrm{yr}^{-1} ; \beta=0.2\right.$; $v_{\exp }(\mathrm{OH})=3 \mathrm{~km} \mathrm{~s}^{-1}$ ) coupled with $[\mathrm{Rb} / \mathrm{Fe}]<0.7$ dex (see Fig. 9).

Figure 10 displays the $[\mathrm{Rb} / \mathrm{Fe}]$ abundances from the best spectral fits versus the variability periods $P$. As mentioned above, our sample is composed of AGB stars of different progenitor masses and evolutionary stages. Most stars with $P>$ 400 days are Li-rich and present some $\mathrm{Rb}$ enhancement ${ }^{6}$, which suggests that, on average, these stars are more massive stars experiencing $\mathrm{HBB}$ and/or more evolved stars (because of the longer periods) than the group of non Li-rich (and generally Rbpoor) stars with $P<400$ days. The stars IRAS 05027-2158 $(P=368$ days $)$ and IRAS 20343-3020 ( $P=349$ days $)$ are exceptions in the latter group. IRAS $05027-2158$ is slightly $\mathrm{Li}$-rich and $\mathrm{Rb}$-poor, suggesting that it is a relatively massive

6 The only exceptions are IRAS 04404-7427 and IRAS 19059-2219, whose optical counterparts are too red to estimate their Li abundances (i.e. the $\mathrm{S} / \mathrm{N}$ at $6708 \AA$ is too low; see García-Hernández et al. 2007). 
Table 3. Atmosphere parameters and best-fit Rb pseudo-dynamical abundances for the listed wind parameters $\dot{M}$ and $\beta$.

\begin{tabular}{ccccccccc}
\hline \hline IRAS name & $T_{\text {eff }}(\mathrm{K})$ & $\log g$ & $\beta$ & $\dot{M}\left(M_{\odot} \mathrm{yr}^{-1}\right)$ & $v_{\text {exp }}(\mathrm{OH})\left(\mathrm{km} \mathrm{s}^{-1}\right)$ & {$[\mathrm{Rb} / \mathrm{Fe}]_{\mathrm{static}}$} & {$[\mathrm{Rb} / \mathrm{Fe}]_{\text {dyn }}$} & {$[\mathrm{Zr} / \mathrm{Fe}]_{\text {dyn }}$} \\
\hline $01085+3022$ & $3000^{*}$ & -0.5 & 0.2 & $1.0 \times 10^{-7}$ & 13 & 2.0 & 0.6 & $\leq 0.0$ \\
$04404-7427$ & 3000 & -0.5 & 0.2 & $1.0 \times 10^{-7}$ & 8 & 1.3 & 0.1 & $\leq 0.0$ \\
$05027-2158$ & 2800 & -0.5 & 0.4 & $1.0 \times 10^{-7}$ & 8 & 0.4 & -0.7 & $\leq+0.5$ \\
$05098-6422$ & 3000 & -0.5 & 1.4 & $1.0 \times 10^{-8}$ & 6 & 0.1 & -0.2 & $\leq+0.25$ \\
$05151+6312$ & 3000 & -0.5 & 1.0 & $1.0 \times 10^{-8}$ & 15 & 2.1 & 1.3 & $\leq+0.25$ \\
$06300+6058$ & 3000 & -0.5 & 0.2 & $1.0 \times 10^{-7}$ & 12 & 1.6 & 0.4 & $\leq 0.0$ \\
$07222-2005$ & 3000 & -0.5 & $\ldots$ & $\ldots$ & 8 & 0.6 & $\ldots$ & $\ldots$ \\
$09194-4518$ & 3000 & -0.5 & $\ldots$ & $\ldots$ & 11 & 1.1 & $\ldots$ & $\ldots$ \\
$10261-5055$ & 3000 & -0.5 & 0.2 & $1.0 \times 10^{-9}$ & 4 & $<-1.0$ & $<-1.1$ & $\leq+0.25$ \\
$14266-4211$ & 2900 & -0.5 & 0.2 & $5.0 \times 10^{-8}$ & 9 & 0.9 & 0.1 & $\leq 0.0$ \\
$15193+3132$ & 2800 & -0.5 & 1.6 & $1.0 \times 10^{-9}$ & 3 & -0.3 & -0.5 & $\leq 0.0$ \\
$15576-1212$ & 3000 & -0.5 & 0.2 & $1.0 \times 10^{-8}$ & 10 & 1.5 & 1.0 & $\leq 0.0$ \\
$16030-5156$ & 3000 & -0.5 & 0.2 & $1.0 \times 10^{-8}$ & 10 & 1.3 & 0.6 & $\leq+0.25$ \\
$16037+4218$ & 2900 & -0.5 & 1.2 & $1.0 \times 10^{-8}$ & 4 & 0.6 & 0.2 & $\leq+0.25$ \\
$17034-1024$ & 3300 & -0.5 & 0.8 & $1.0 \times 10^{-8}$ & 8 & 0.2 & -0.7 & $\leq 0.0$ \\
$18429-1721$ & 3000 & -0.5 & 0.2 & $1.0 \times 10^{-8}$ & 7 & 1.2 & 0.9 & $\leq+0.25$ \\
$19059-2219$ & 3000 & -0.5 & 0.2 & $1.0 \times 10^{-7}$ & 13 & 2.3 & 0.5 & $\leq+0.25$ \\
$19426+4342$ & 3000 & -0.5 & $\ldots$ & $\ldots$ & 9 & 1.0 & $\ldots$ & $\ldots$ \\
$20052+0554$ & $3000 *$ & -0.5 & 0.2 & $5.0 \times 10^{-7}$ & 16 & 1.5 & 0.0 & $\leq 0.0$ \\
$20077-0625$ & 3000 & -0.5 & $\ldots$ & $\ldots$ & 12 & 1.3 & $\ldots$ & $\ldots$ \\
$20343-3020$ & 3000 & -0.5 & 1.2 & $1.0 \times 10^{-9}$ & 8 & 0.9 & 0.7 & $\leq 0.0$ \\
\hline
\end{tabular}

Notes. The asterisks indicate that the best fitting $T_{\text {eff }}$ in the $\mathrm{ZrO} 6474 \AA$ spectral region is warmer $(3300 \mathrm{~K})$ than the one around the $\mathrm{Rb} \mathrm{I} 7800 \AA$ line (García-Hernández et al. 2006, 2007). We have checked the sensitivity of the derived abundances to small changes in the model atmosphere parameters $\left(\Delta T_{\text {eff }}= \pm 100 \mathrm{~K}, \Delta \beta=0.2, \Delta \log \left(\dot{M} / M_{\odot} \mathrm{yr}^{-1}\right)=0.5, \Delta v_{\text {exp }}(\mathrm{OH})=5 \mathrm{~km} \mathrm{~s}^{-1}\right)$. The dominant sources of uncertainties are $\dot{M}$ and $T_{\text {eff }}$ for $\mathrm{Rb}$ and $\mathrm{Zr}$, respectively, which result in error bars of \pm 0.7 and \pm 0.3 dex, respectively. In the hydrostatic case, the formal Rb uncertainties (quoted by García-Hernández et al. 2006) due to changes of all the atmosphere parameters are \pm 0.8 dex.

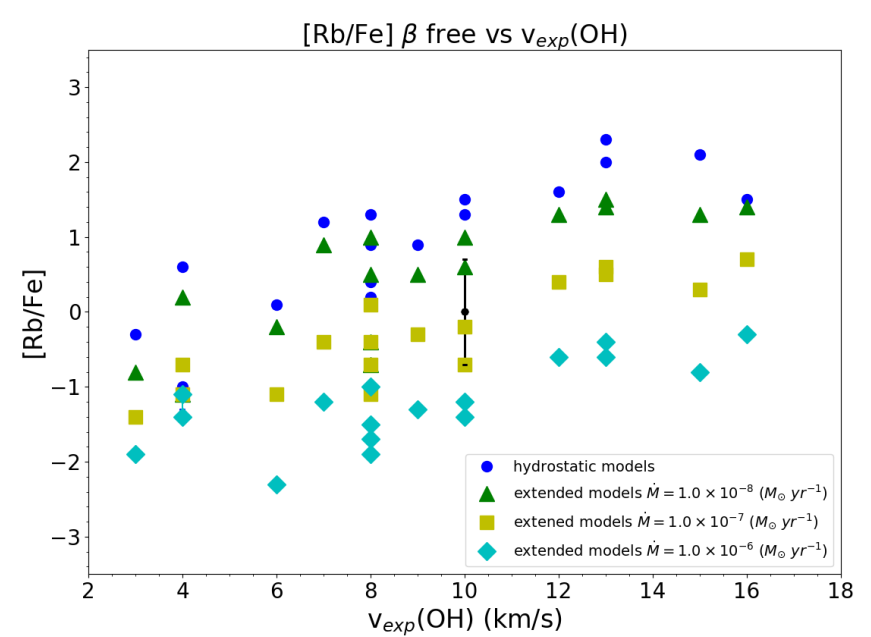

Fig. 8. $\mathrm{Rb}$ abundances vs. the expansion velocity $\left(v_{\exp }(\mathrm{OH})\right)$ for extended model atmospheres with $\dot{M}=10^{-8}, 10^{-7}$ and $10^{-6} M_{\odot} \mathrm{yr}^{-1}$ (green triangles, yellow squares and cyan diamonds, respectively) in comparison with those obtained from hydrostatic models (blue dots).

AGB star (say $\sim 3.5-4.5 M_{\odot}{ }^{7}$ ) at the beginning of the TP phase (e.g. in an inter-pulse period immediately before or after the

\footnotetext{
7 The initial mass for HBB activation is model dependent; i.e. at solar metallicity HBB is activated at $\sim 3.5$ and $4.5 M_{\odot}$ depending on the mass-loss and convection prescriptions used in the models (see e.g. García-Hernández et al. 2013, for more details).
}

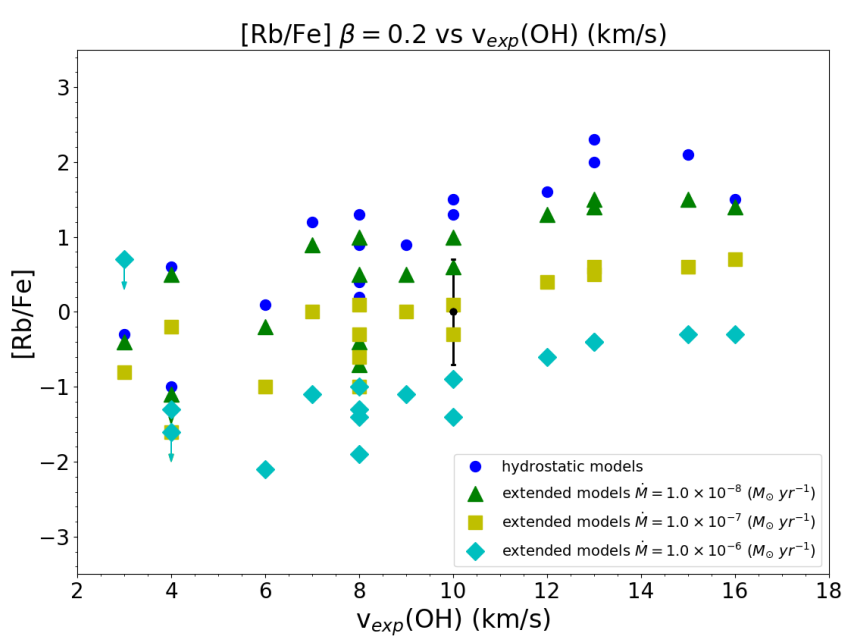

Fig. 9. $\mathrm{Rb}$ abundances vs. the expansion velocity $\left(v_{\exp }(\mathrm{OH})\right)$ for extended model atmospheres with $\beta=0.2$ and $\dot{M}=10^{-8}, 10^{-7}$ and $10^{-6} M_{\odot} \mathrm{yr}^{-1}$ (green triangles, yellow squares and cyan diamonds, respectively) in comparison with those obtained from hydrostatic models (blue dots).

super Li-rich phase) but is not evolved enough for efficient $\mathrm{Rb}$ production (García-Hernández et al. 2013). On the other hand, IRAS 20343-3020 is slightly Rb-rich and Li-poor, which suggests a more advanced evolutionary stage and a slightly higher initial mass (say 4.0-5 $M_{\odot}$ ) than IRAS 05027-2158 (we refer to Fig. 1 in García-Hernández et al. 2013). 
Table 4. Atmosphere parameters and $\mathrm{Rb}$ pseudo-dynamical abundances with $\beta=0.2$ and $\beta=1.2$, respectively.

\begin{tabular}{|c|c|c|c|c|c|c|c|c|}
\hline IRAS name & $T_{\text {eff }}(\mathrm{K})$ & $\log g$ & $\beta$ & $\dot{M}\left(M_{\odot} \mathrm{yr}^{-1}\right)$ & $v_{\exp }(\mathrm{OH})\left(\mathrm{km} \mathrm{s}^{-1}\right)$ & {$[\mathrm{Rb} / \mathrm{Fe}]_{\text {static }}$} & {$[\mathrm{Rb} / \mathrm{Fe}]_{\mathrm{dyn}}$} & {$[\mathrm{Zr} / \mathrm{Fe}]_{\mathrm{dyn}}$} \\
\hline \multirow{2}{*}{$01085+3022$} & \multirow{2}{*}{$3000^{*}$} & \multirow{2}{*}{-0.5} & 0.2 & $1.0 \times 10^{-7}$ & \multirow{2}{*}{13} & \multirow{2}{*}{2.0} & 0.6 & \multirow{2}{*}{$\leq 0.0$} \\
\hline & & & 1.2 & $1.0 \times 10^{-7}$ & & & 0.3 & \\
\hline \multirow{2}{*}{ 04404-7427 } & \multirow{2}{*}{3000} & \multirow{2}{*}{-0.5} & 0.2 & $1.0 \times 10^{-7}$ & \multirow{2}{*}{8} & \multirow{2}{*}{1.3} & 0.1 & \multirow{2}{*}{$\leq 0.0$} \\
\hline & & & 1.2 & $1.0 \times 10^{-7}$ & & & 0.1 & \\
\hline \multirow{2}{*}{$05027-2158$} & \multirow{2}{*}{2800} & \multirow{2}{*}{-0.5} & 0.2 & $1.0 \times 10^{-7}$ & \multirow{2}{*}{8} & \multirow{2}{*}{0.4} & -0.6 & \multirow{2}{*}{$\leq+0.5$} \\
\hline & & & 1.2 & $1.0 \times 10^{-7}$ & & & -1.1 & \\
\hline \multirow{2}{*}{$05098-6422$} & \multirow{2}{*}{3000} & \multirow{2}{*}{-0.5} & 0.2 & $1.0 \times 10^{-8}$ & \multirow{2}{*}{6} & \multirow{2}{*}{0.1} & -0.2 & \multirow{2}{*}{$\leq+0.25$} \\
\hline & & & 1.2 & $5.0 \times 10^{-7}$ & & & -2.1 & \\
\hline \multirow{2}{*}{$05151+6312$} & 3000 & -0.5 & 0.2 & $5.0 \times 10^{-7}$ & 15 & 2.1 & 0.0 & $<+0.25$ \\
\hline & & -0.5 & 1.2 & $5.0 \times 10^{-7}$ & 15 & 2.1 & -0.5 & $-+0.2 J$ \\
\hline $06300+6058$ & 3000 & -0.5 & 0.2 & $1.0 \times 10^{-7}$ & 12 & 16 & 0.4 & $<00$ \\
\hline $00500+0050$ & 3000 & -0.5 & 1.2 & $1.0 \times 10^{-7}$ & 12 & 1.0 & 0.3 & $\leq 0.0$ \\
\hline $07222-2005$ & 3000 & -0.5 & $\ldots$ & $\ldots$ & 8 & 0.6 & $\ldots$ & $\ldots$ \\
\hline 09194-4518 & 3000 & -0.5 & $\ldots$ & $\ldots$ & 11 & 1.1 & $\ldots$ & $\ldots$ \\
\hline $10261-5055$ & 3000 & -05 & 0.2 & $1.0 \times 10^{-9}$ & 4 & $<-10$ & $<-1.1$ & $<+025$ \\
\hline $10201-5055$ & 3000 & -0.5 & 1.2 & $1.0 \times 10^{-9}$ & 4 & $<-1.0$ & $<-1.1$ & $\leq+0.25$ \\
\hline $14266-4211$ & 2000 & -05 & 0.2 & $5.0 \times 10^{-8}$ & 9 & 09 & 0.1 & $\leq 0.0$ \\
\hline $14200-4211$ & 2900 & -0.5 & 1.2 & $1.0 \times 10^{-7}$ & 9 & 0.9 & -0.4 & $\leq-0.25$ \\
\hline $15193+3132$ & 2800 & -0.5 & 0.2 & $1.0 \times 10^{-9}$ & 3 & -03 & -0.4 & $<00$ \\
\hline 2018 & & -0.5 & 1.2 & $1.0 \times 10^{-9}$ & $J$ & -0.3 & -0.4 & $\leq 0.0$ \\
\hline $15576-1212$ & 3000 & -05 & 0.2 & $1.0 \times 10^{-8}$ & 10 & 15 & 1.0 & $<00$ \\
\hline $153 / 0-1212$ & 3000 & -0.5 & 1.2 & $1.0 \times 10^{-8}$ & 10 & 1.5 & 0.9 & $\leq 0.0$ \\
\hline $16030-5156$ & 3000 & -0.5 & 0.2 & $1.0 \times 10^{-8}$ & 10 & 13 & 0.6 & $<+025$ \\
\hline & & 0.0 & 1.2 & $1.0 \times 10^{-7}$ & 10 & & -0.4 & -+0.20 \\
\hline $16037+4218$ & 2900 & -05 & 0.2 & $1.0 \times 10^{-8}$ & 4 & 06 & 0.5 & $<+025$ \\
\hline $10031+4218$ & 2900 & -0.5 & 1.2 & $1.0 \times 10^{-8}$ & 4 & 0.0 & 0.2 & $\leq+0.20$ \\
\hline 17034-1024 & 3300 & -05 & 0.2 & $1.0 \times 10^{-8}$ & 8 & 02 & -0.7 & $<00$ \\
\hline 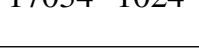 & 3500 & -0.5 & 1.2 & $1.0 \times 10^{-8}$ & 0 & & -0.8 & $\leq 0.0$ \\
\hline $18429-1721$ & 3000 & -0.5 & 0.2 & $1.0 \times 10^{-8}$ & 7 & 1.2 & 0.9 & $\leq+0.25$ \\
\hline & & -0.5 & 1.2 & $5.0 \times 10^{-7}$ & 1 & 1.2 & -1.0 & $\leq 0.0$ \\
\hline $19059-2219$ & 3000 & $-0,5$ & 0.2 & $1.0 \times 10^{-7}$ & 13 & 23 & 0.5 & 25 \\
\hline $19059-2219$ & 3000 & -0.5 & 1.2 & $5.0 \times 10^{-8}$ & 13 & 2.5 & 0.5 & $=+0.25$ \\
\hline $19426+4342$ & 3000 & -0.5 & $\ldots$ & $\ldots$ & 9 & 1.0 & $\ldots$ & $\ldots$ \\
\hline $20052+0554$ & $3000 *$ & -05 & 0.2 & $5.0 \times 10^{-7}$ & 16 & 15 & 0.0 & $<00$ \\
\hline $200 J 2+0304$ & 3000 & -0.0 & 1.2 & $5.0 \times 10^{-7}$ & 10 & 1.0 & -0.7 & -0.0 \\
\hline $20077-0625$ & 3000 & -0.5 & $\ldots$ & $\ldots$ & 12 & 1.3 & $\ldots$ & $\ldots$ \\
\hline $20343-3020$ & 3000 & -05 & 0.2 & $1.0 \times 10^{-9}$ & 8 & 09 & 0.7 & $<00$ \\
\hline 20020 & 3000 & 0.0 & 1.2 & $1.0 \times 10^{-9}$ & 0 & 0.3 & 0.7 & -0.0 \\
\hline
\end{tabular}

Notes. The asterisks indicate that the best fitting $T_{\text {eff }}$ in the $\mathrm{ZrO} 6474 \AA$ spectral region is warmer $(3300 \mathrm{~K})$ than the one around the Rb I $7800 \AA$ line (García-Hernández et al. 2006, 2007).

\section{Comparison with AGB nucleosynthesis models}

In Fig. 11 we compare our new $[\mathrm{Rb} / \mathrm{Fe}]$ abundances and $[\mathrm{Rb} / \mathrm{Zr}]$ ratios with solar metallicity massive $\left(3-9 M_{\odot}\right)$ AGB predictions from several nucleosynthesis models: van Raai et al. (2012), Karakas et al. (2012), Karakas \& Lugaro (2016) (Monash), Pignatari et al. (2016) (NuGrid/MESA) and Cristallo et al. (2015) (FRUITY ${ }^{8}$ ). The predicted $[\mathrm{Rb} / \mathrm{Fe}]$ abundances and $[\mathrm{Rb} / \mathrm{Zr}]$ ratio ranges are $0.00-1.35$ and $-0.45-0.52$ dex, respectively.

The Monash models (van Raai et al. 2012; Karakas et al. 2012; Karakas \& Lugaro 2016) use the stellar evolutionary

8 FUll-Network Repository of Updated Isotopic Tables and Yields: http://fruity.oa-teramo.inaf.it/ 


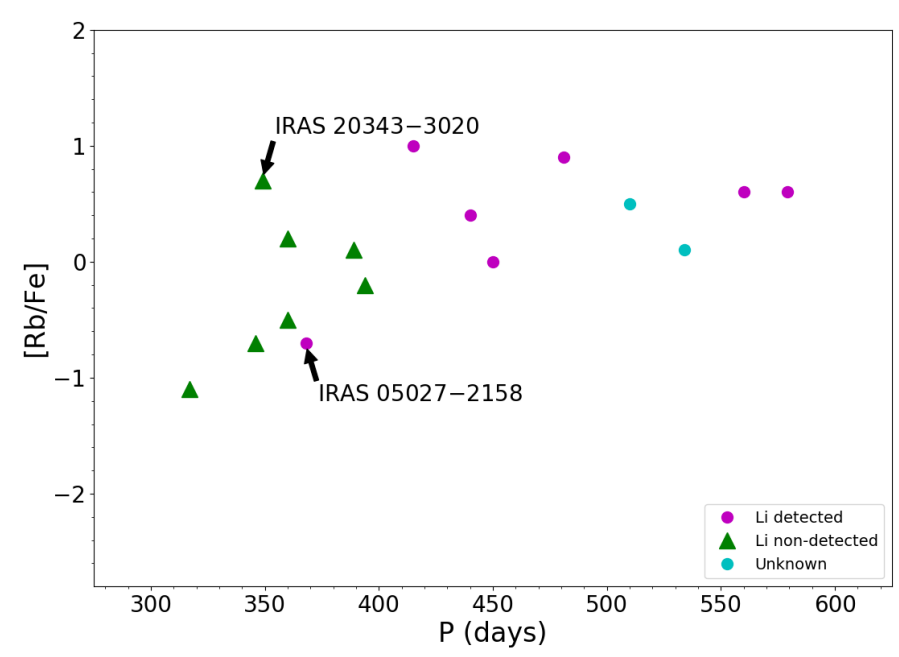

Fig. 10. $[\mathrm{Rb} / \mathrm{Fe}]$ pseudo-dynamical abundances versus variability period $(P)$. The Li-rich and Li-poor stars are marked with magenta dots and green triangles, respectively. The two stars where Li could not be estimated are marked with cyan dots (see text).

sequences calculated with the Monash version of the Mount Stromlo Stellar Structure Program (Frost \& Lattanzio 1996), which uses the Vassiliadis \& Wood (1993) mass-loss prescription on the AGB. A post-processing code is used to obtain in detail the nucleosynthesis of a large number of species, including the $s$-process abundances. Due to convergence difficulties, the stellar evolution models used in the calculations are not always evolved until the end of the superwind phase and synthetic models have been used to estimate the effect of remaining TPs and to completely remove the envelope. We refer the reader to van Raai et al. (2012), Karakas et al. (2012) and Karakas \& Lugaro (2016) for more details about the theoretical models. Here, we only report on the main differences between these models: i) The use of different nuclear networks; that is, the total number of nuclear species considered and the values of some reaction rates and neutron-capture cross-sections (see below); and ii) the use by Karakas et al. (2012) of a modified Vassiliadis \& Wood (1993) mass-loss prescription, which delays the beginning of the superwind phase until the pulsation period reaches values of 700-800 days (instead of the value of 500 days used in the other models), resulting in a higher $\mathrm{Rb}$ production.

The NuGrid/MESA and FRUITY models assume AGB mass-loss prescriptions, nuclear physics inputs and treatments of convection different from the Monash models. In particular, the Blöcker (1995) and Straniero, Gallino \& Cristallo (2006) mass-loss formulae for the AGB phase are assumed by the NuGrid/MESA and FRUITY models, respectively. Furthermore, these models produce self-consistently the ${ }^{13} \mathrm{C}$ neutron source as a result of the different convective boundary mixing scheme and treatments of the convective borders, while in the Monash models the mixing required to produce the ${ }^{13} \mathrm{C}$ neutron source is included in a parametrised way during the post processing and is typically not included in massive AGB stars, following theoretical (Goriely \& Siess 2004) and observational indications (García-Hernández et al. 2013) (we refer also to Pignatari et al. 2016; Cristallo et al. 2015; Karakas \& Lugaro 2016, for more details). In relation to the main results, i) the NuGrid/MESA solar metallicity massive AGB models are qualitatively similar to the Monash models in that HBB and light s-process element production (of the elements from $\mathrm{Rb}$ to $\mathrm{Zr}$ ) are seen at the stellar surface, the latter due to the activation of the ${ }^{22} \mathrm{Ne}$ neutron source and the subsequent operation of the TDU; and ii) the FRUITY solar metallicity massive AGB models are different to the Monash and NuGrid/MESA models because these models experience very inefficient TDU, hence the signature of the nucleosynthesis due to the ${ }^{22} \mathrm{Ne}$ neutron source is not visible at the stellar surface.

Figure 11 shows that the FRUITY massive AGB model predicts final $[\mathrm{Rb} / \mathrm{Fe}]<0.15$, which does not explain the observed range of $\mathrm{Rb}$ abundances and $[\mathrm{Rb} / \mathrm{Zr}]$ ratios; specifically the $[\mathrm{Rb} / \mathrm{Zr}]$ ratios remain negative for all masses. Another difference between the FRUITY models and the Monash and NuGrid models is that the FRUITY models do not predict $\mathrm{HBB}$ to occur in AGB stars unless the metallicity is very low; at least ten times lower than solar. However, spectroscopic observations of massive AGB stars demonstrate that they experience $\mathrm{HBB}$; as evidenced by i) strong $\mathrm{Li}$ overabundances observed in massive AGB stars in the Galaxy (Fe/H = 0.0; e.g. García-Hernández et al. 2007, 2013), the Magellanic Clouds ( $\mathrm{Fe} / \mathrm{H}=-0.7-0.3$; e.g. Plez et al. 1993; Smith et al. 1995; García-Hernández et al. 2009) and the dwarf galaxy IC $1613(\mathrm{Fe} / \mathrm{H}=-1.6$; e.g. Menzies, Whitelock \& Feast 2015); and ii) $\mathrm{N}$ enhancements and low ${ }^{12} \mathrm{C} /{ }^{13} \mathrm{C}$ ratios in Magellanic Cloud Li-rich massive AGBs (e.g. Plez et al. 1993; McSaveney et al. 2007). The lack of HBB in the FRUITY predictions is also at odds with the observations of the so-called type-I planetary nebulae in very different metallicity environments and galaxies; which are expected to be the descendants of HBB massive AGB stars based on their strong $\mathrm{N}$ and $\mathrm{He}$ overabundances (see e.g. Stanghellini et al. 2006; Karakas et al. 2009; Leisy\& Dennefeld 1996; García-Rojas et al. 2016, and references therein).

It is to be noted here that the several Monash AGB models (van Raai et al. 2012; Karakas et al. 2012; Karakas \& Lugaro 2016) mentioned above notably use different rates for the ${ }^{22} \mathrm{Ne}(\alpha, n){ }^{25} \mathrm{Mg}$ reaction, which drives the production of $s$-process elements in massive AGB stars. In particular, Karakas \& Lugaro (2016) use the ${ }^{22} \mathrm{Ne}(\alpha, n)^{25} \mathrm{Mg}$ reaction from Iliadis et al. (2010), neutron-capture cross-section of the $\mathrm{Zr}$ isotopes (Lugaro et al. 2014), and a more extended nuclear network of 328 species (from $\mathrm{H}$ to $\mathrm{S}$, and then from $\mathrm{Fe}$ to $\mathrm{Bi}$ ). The van Raai et al. (2012) models, instead, use a nuclear network of 166 species (up to $\mathrm{Nb}$ ) and the ${ }^{22} \mathrm{Ne}(\alpha, n)^{25} \mathrm{Mg}$ reaction rate from Karakas et al. (2006), while Karakas et al. (2012) explored different networks (166, 172 and 320 species) and ${ }^{22} \mathrm{Ne}(\alpha, n)^{25} \mathrm{Mg}$ reaction rates; from Karakas et al. (2006), Iliadis et al. (2010) and Angulo et al. (1999), NACRE.

The van Raai et al. (2012) models (from 4 to $6.5 M_{\odot}$ at $Z=0.02$; Fig. 11) show that both the $[\mathrm{Rb} / \mathrm{Fe}]$ abundances and $[\mathrm{Rb} / \mathrm{Zr}]$ ratios increase with the initial mass of the AGB star, as the star becomes hotter and the ${ }^{22} \mathrm{Ne}(\alpha, n)^{25} \mathrm{Mg}$ reaction is more efficiently activated. However, the $[\mathrm{Rb} / \mathrm{Fe}]$ abundances from the last computed TP are too low (ranging from 0.0 to $0.26 \mathrm{dex}$ ). The corresponding $\mathrm{Rb}$ abundances $([\mathrm{Rb} / \mathrm{Fe}] \sim 0.0-1.0 \mathrm{dex}$ ) from the synthetic evolution calculations cover most of the Rb abundances observed; although they cannot explain the star IRAS $05151+6312$ with $[\mathrm{Rb} / \mathrm{Fe}]=1.3 \mathrm{dex}$. Such high $\mathrm{Rb}$ abundances can be reached by the synthetic calculations of the solar metallicity 6 and $7 M_{\odot}$ AGB models with delayed superwinds of Karakas et al. (2012) when using the faster NACRE rate for the ${ }^{22} \mathrm{Ne}(\alpha, n)^{25} \mathrm{Mg}$ reaction. Finally, the Karakas \& Lugaro (2016) models (from 4.5 to $8 M_{\odot}$ at $Z=0.014{ }^{9}$; Fig. 11) predict lower

9 According to the more recent solar abundances from Asplund et al. (2009). 

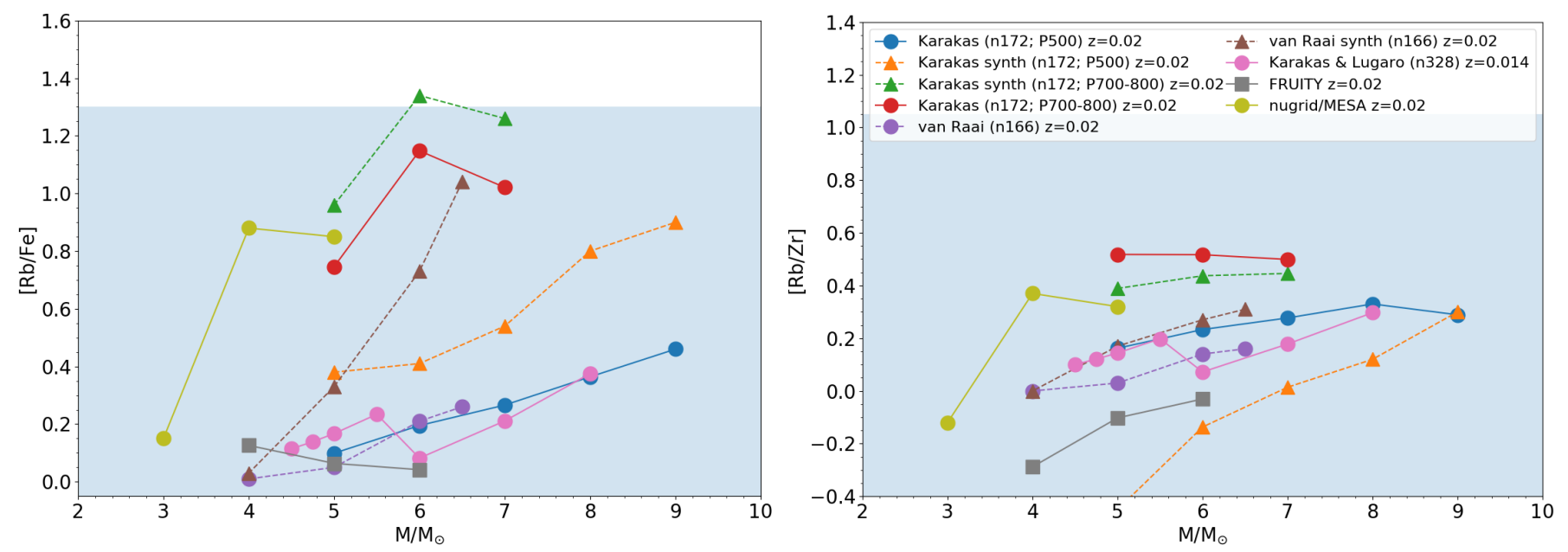

Fig. 11. Model predictions from van Raai et al. (2012), Karakas et al. (2012), Karakas \& Lugaro (2016), FRUITY database and Pignatari et al. (2016): Stellar mass vs. [Rb/Fe] (left panel) and [Rb/Zr] (right panel). The abundances from the last computed thermal pulse and from the synthetic evolution calculations are shown with dots and triangles, respectively. $P$ represents the period for the beginning of the superwind phase and $n$ is the number of species in the nucleosynthesis network. The shaded regions mark the range of the new $\mathrm{Rb}$ abundances and [Rb/Zr] ratios obtained in our sample with extended models.

Rb abundances than the Karakas et al. (2012) models of the same mass and similar metallicity, mostly due to the implementation of the delayed superwind and the use of the NACRE rate in Karakas et al. (2012).

The NuGrid/MESA models (from 3 to $5 M_{\odot}$ at $Z=0.02$; Fig. 11) reproduce the observed $[\mathrm{Rb} / \mathrm{Fe}]$ and $[\mathrm{Rb} / \mathrm{Zr}]$ ranges, up to 0.9 and 0.4 dex, respectively. However, we note that only in the $5 M_{\odot}$ case do the NuGrid/MESA models see signature of HBB and predict O-rich stars. The 3 and $4 M_{\odot}$ cases become C-rich stars and do not experience HBB, which is at odds with our sample of O-rich stars (García-Hernández et al. 2006).

Regarding the $[\mathrm{Rb} / \mathrm{Zr}]$ ratios, obviously also in this case the higher $[\mathrm{Rb} / \mathrm{Zr}]$ ratios are obtained from the models with delayed superwind $(P=700-800)$, however, these $[\mathrm{Rb} / \mathrm{Zr}]$ ratios are still lower than our observed values. The maximum value from the AGB models is $[\mathrm{Rb} / \mathrm{Zr}]=0.52$ for $M=5 M_{\odot}$, and the maximum value from our observations is $[\mathrm{Rb} / \mathrm{Zr}]=1.05$. A possible explanation is that $\mathrm{Zr}$ could be depleted into dust (e.g. van Raai et al. 2012; Zamora et al. 2014), producing the differences between the theoretical and observational $[\mathrm{Rb} / \mathrm{Zr}]$ ratios. Abundance measurements of similar s-elements such as $\mathrm{Sr}$ and $\mathrm{Y}$ would be needed in order to clarify this problem.

\section{Conclusions}

Here we report new $\mathrm{Rb}$ and $\mathrm{Zr}$ abundances determined from the $7800 \AA \mathrm{Rb}$ I line and the $6474 \AA \mathrm{ZrO}$ bandhead, respectively, in a complete sample of massive Galactic AGB stars, previously studied with hydrostatic models, using more realistic extended atmosphere models and a modified version of the spectral synthesis code Turbospectrum, which considers the presence of a circumstellar envelope with a radial wind. The Rb abundances are much lower (in some cases even 1-2 dex) with the pseudo-dynamical models, while the $\mathrm{Zr}$ abundances are close to the hydrostatic ones because the $6474 \AA \mathrm{ZrO}$ bandhead is formed deeper in the atmosphere and is less affected than the $7800 \AA \mathrm{Rb}$ I resonant line by the circumstellar effects.

We have studied the sensitivity of the determined abundances to variations in the stellar $\left(T_{\text {eff }}\right)$ and wind $\left(\dot{M}, \beta\right.$ and $\left.v_{\text {exp }}\right)$ parameters. The $\mathrm{Rb}$ abundances are very sensitive to the mass loss rate
$\dot{M}$ but much less to the $\beta$ parameter and $v_{\text {exp }}(\mathrm{OH})$. The $\mathrm{Zr}$ abundances, instead, are not affected by variations of the stellar and wind parameters. The $\mathrm{Rb}$ abundances from extended models are lower than those obtained from the hydrostatic ones, and the difference is larger in the stars with the highest $\mathrm{Rb}$ abundances in the hydrostatic case. We have represented the hydrostatic and pseudo-dynamical $\mathrm{Rb}$ abundances against the $v_{\text {exp }}(\mathrm{OH})$, which can be used as a mass indicator independent of the distance, and we have observed a flatter correlation. The difference between the hydrostatic and pseudo-dynamical $\mathrm{Rb}$ abundances increases with increasing the $v_{\exp }(\mathrm{OH})$, due to the fact that the presence of a circumstellar envelope affects more strongly the more massive stars. Furthermore, the dispersion of the correlation between the $\mathrm{Rb}$ abundance and $v_{\text {exp }}(\mathrm{OH})$ is larger in the pseudo-dynamical case. When we fix the wind parameters $\dot{M}$ (i.e. equivalent to assuming that our AGB sample stars have a similar evolutionary stage in terms of mass loss), and/or $\beta$ (the same velocity profile), the dispersion is lower.

The Monash nucleosynthesis theoretical predictions reproduce the range of new $\mathrm{Rb}$ and $\mathrm{Zr}$ abundances although $[\mathrm{Rb} / \mathrm{Fe}]$ values above 1.0 can only be matched if the superwind is delayed to after the period reaches 700-800 days. We also note that the rate of the ${ }^{22} \mathrm{Ne}(\alpha, n)^{25} \mathrm{Mg}$ reaction is crucial, but still hampered by large systematic uncertainties (e.g. Bisterzo et al. 2016; Massimi et al. 2017). Underground measurements, planned, for example, at LNGS-LUNA (Laboratory for Underground $\mathrm{Nu}$ clear Astrophysics) will help to resolve the current issues. The FRUITY massive AGB models predict $\mathrm{Rb}$ abundances much lower than observed and negative $[\mathrm{Rb} / \mathrm{Zr}]$ ratios, at odds with the observations. The NuGrid/MESA models of 4 and $5 M_{\odot}$ predict $[\mathrm{Rb} / \mathrm{Fe}]$ as high as $0.9 \mathrm{dex}$, however, the $4 M_{\odot}$ model does not experience HBB and becomes C-rich, while our sample stars are clearly O-rich. The maximum observed $[\mathrm{Rb} / \mathrm{Zr}]$ ratios are still more than a factor of two larger than predicted by the nucleosynthesis models. A possible explanation for this difference between the observations and the predictions is that $\mathrm{Zr}$ could be depleted into dust. Observations of other $s$-process elements $\mathrm{Sr}$ and $\mathrm{Y}$ belonging to the same first peak as $\mathrm{Rb}$ and $\mathrm{Zr}$ will help to clarify this mismatch.

In summary, the $[\mathrm{Rb} / \mathrm{Fe}]$ abundances and $[\mathrm{Rb} / \mathrm{Zr}]$ ratios previously derived with hydrostatic models are certainly not 
predicted by the most recent theoretical models of AGB nucleosynthesis. In particular, the highest $[\mathrm{Rb} / \mathrm{Fe}]$ abundances and $[\mathrm{Rb} / \mathrm{Zr}]$ ratios observed in massive Galactic AGBs are much larger than theoretically predicted. The new $[\mathrm{Rb} / \mathrm{Fe}]$ abundances and $[\mathrm{Rb} / \mathrm{Zr}]$ ratios as obtained from our simple (but more realistic) pseudo-dynamical model atmospheres are much lower in much better agreement with the theoretical predictions, significantly resolving the mismatch between the observations and the nucleosynthesis models in the more massive AGB stars. This confirms the earlier Zamora et al. preliminary results on a smaller sample of massive O-rich AGB stars but here we find that the $\mathrm{Rb}$ abundances are strongly dependent on the wind mass-loss $\dot{M}$, which is basically unknown in our AGB star sample. Followup radio observations (e.g. the rotational lines of the several $\mathrm{CO}$ isotopologues) of these massive Galactic AGB stars are encouraged in order to obtain precise mass-loss rate estimates needed to break the actual models degeneracy and obtain more reliable (non-model-dependent) Rb abundances in massive AGB stars.

Acknowledgements. This work is based on observations at the $4.2 \mathrm{~m}$ William Herschel Telescope operated on the island of La Palma by the Isaac Newton Group in the Spanish Observatorio del Roque de Los Muchachos of the Instituto de Astrofisica de Canarias. Also based on observations with the ESO $3.6 \mathrm{~m}$ telescope at La Silla Observatory (Chile). We thank Marco Pignatari and Umberto Battino for providing information about the Nugrid/MESA models. V.P.M. acknowledges the financial support from the Spanish Ministry of Economy and Competitiveness (MINECO) under the 2011 Severo Ochoa Program MINECO SEV-2011-0187. D.A.G.H. was funded by the Ramón y Cajal fellowship number RYC-2013-14182. V.P.M., O.Z., D.A.G.H. and A.M. acknowledge support provided by the MINECO under grant AYA-2014-58082-P. M.L. is a Momentum ("Lendület-2014" Programme) project leader of the Hungarian Academy of Sciences. M.L. acknowledges the Instituto de Astrofísica de Canarias for inviting her as a Severo Ochoa visitor during 2015 August when part of this work was done. This paper made use of the IAC Supercomputing facility HTCondor (http://research.cs.wisc.edu/htcondor/), partly financed by the Ministry of Economy and Competitiveness with FEDER funds, code IACA13-3E2493. This work benefited from discussions at The 12th Torino Workshop on Asymptotic Giant Branch Stars in August 2016 supported by the National Science Foundation under Grant No. PHY-1430152 (JINA Center for the Evolution of the Elements).

\section{References}

Abia, C., Busso, M., Gallino, R., et al. 2001, ApJ, 559, 1117

Alvarez, R., \& Plez, B. 1998, A\&A, 330, 1109

Angulo, C., Arnould, M., Rayet, M., et al. 1999, Nucl. Phys. A, 656, 3

Asplund, M., Grevesse, N., Sauval, A. J., \& Scott, P. 2009, ARA\&A, 47, 481

Beer, H., \& Macklin, R. L. 1989, ApJ, 339, 962

Bisterzo, S., Travaglio, C., Wiescher, M., et al. 2016, J. Phys. Conf. Ser., 665 012023

Busso, M., Gallino, R., \& Wasserburg, G. J. 1999, ARA\&A, 37, 239

Blöcker, T. 1995, A\&A, 297, 727

Cristallo, S., Piersanti, L., Straniero, O., et al. 2011, ApJS, 197, 17

Cristallo, S., Straniero, O., Piersanti, L., \& Gobrecht, D. 2015, ApJS, 219, 40

Danilovich, T., Teyssier, D., Justtanont, K. et al. 2015, A\&A, 581, A60

De Beck, E., Decin, L., de Koter, A., et al. 2010, A\&A, 523, A18

Decin, L., Justtanont, K., De Beck, E., et al. 2010, A\&A, 521, L4
Di Criscienzo, M., Ventura, P., García-Hernández, D. A., et al. 2016, MNRAS, 462, 395

Frost, C. A., \& Lattanzio, J. C. 1996, ApJ, 473, 383

García-Hernández, D. A., García-Lario, P., Plez, B., et al. 2006, Science, 314, 1751

García-Hernández, D. A., García-Lario, P., Plez, B., et al. 2007, A\&A, 462, 711

García-Hernández, D. A., Manchado, A., Lambert, D. L., et al. 2009, ApJ, 705, L31

García-Hernández, D. A., Zamora, O., Yagüe, A., et al. 2013, A\&A, 555, L3

García-Rojas, J., Peña, M., Flores-Durán, S., \& Hernández-Martínez, L., 2016, A\&A, 586, A59

Goriely, S., \& Siess, L. 2004, A\&A, 421, L25

Grevesse, N., \& Sauval, A. J. 1998, Space Sci. Rev., 85, 161

Grevesse, N., Asplund, M., \& Sauval, A. J. 2007, Space Sci. Rev., 130, 105

Gustafsson, B., Edvardsson, B., Eriksson, K., et al. 2008, A\&A, 486, 951

Herwig, F. 2005, ARA\&A, 43, 435

Hoppe, P., \& Ott, U. 1997, AIP Conf. Ser., 402, 27

Iliadis, C., Longland, R., Champagne, A. E., \& Coc, A. 2010, Nucl. Phys. A, 841,323

Justtanont, K., Teyssier, D., Barlow, M. J., et al. 2013, A\&A, 556, A101

Karakas, A. I. 2014, MNRAS, 445, 347

Karakas, A., \& Lattanzio, J. C. 2007, PASA, 24, 103

Karakas, A. I., \& Lattanzio, J. C. 2014, PASA, 31, e030

Karakas, A. I., \& Lugaro, M. 2016, ApJ, 825, 26

Karakas, A. I., Lugaro, M. A., Wiescher, M., Görres, J., \& Ugalde, C. 2006, ApJ, 643,471

Karakas, A. I., van Raai, M. A., Lugaro, M., Sterling, N. C., Dinerstein, H. L. 2009, ApJ, 690, 1130

Karakas, A. I., Campbell, S. W., \& Stancliffe, R. J. 2010, ApJ, 713, 374

Karakas, A. I., García-Hernández, D. A., \& Lugaro, M. 2012, ApJ, 751, 8

Lambert, D. L., Smith, V. V., Busso, M., Gallino, R., \& Straniero, O. 1995, ApJ, 450,302

Leisy, P., \& Dennefeld, M. 1996, A\&AS, 116, 95

Lugaro, M., \& Chieffi, A. 2011, Lect. Notes Phys. (Berlin: Springer Verlag), 812, 83

Lugaro, M., Karakas, A. I., Stancliffe, R. J., \& Rijs, C. 2012, ApJ, 747, 2

Lugaro, M., Tagliente, G., Karakas, A. I., et al. 2014, ApJ, 780, 95

Lugaro, M., Karakas, A. I., Bruno, C. G., et al. 2017, Nat. Astron., 1, 0027

Massimi, C., Altstadt, S., Andrzejewski, J., et al. 2017, Phys. Lett. B, 768, 1

Mazzitelli, I., D’Antona, F., \& Ventura, P. 1999, A\&A, 348, 846

McSaveney, J. A., Wood, P. R., Scholz, M., Lattanzio, J. C., Hinkle, K. H. 2007, MNRAS, 378, 1089

Menzies, J. W., Whitelock, P. A., \& Feast, M. W. 2015, MNRAS, 452, 910

Nittler, L. R., Alexander, O., Gao, X., Walker, R. M., \& Zinner, E. 1997, ApJ 483,475

Nordlund, A. 1984, in Methods in Radiative Transfer, ed. W. Kalkofen (Cambridge, New York: Cambridge University Press), 211

Pignatari, M., Herwig, F., Hirschi, R. et al. (2016, ApJS, 225, 24

Plez, B. 2012, Astrophysics Source Code Library [record ascl : 1205 . 004]

Plez, B., Smith, V. V., \& Lambert, D. L. 1993, ApJ, 418, 812

Sackmann, I.-J., \& Boothroyd, A. I. 1992, ApJ, 392, L71

Samus, N. N., Durlevich, O. V., \& et al. 2009, VizieR Online Data Catalog B/gcvs

Smith, V. V., Plez, B., Lambert, D. L., Lubowich, D. A. 1995, ApJ, 441, 735

Stanghellini, L., Guerrero, M. A., Cunha, K., Manchado, A., Villaver, E. 2006, ApJ, 651, 898

Straniero, O., Gallino, R. \& Cristallo, S. 2006, Nucl. Phys. A, 777, 311

van Raai, M. A., Lugaro, M., Karakas, A. I., García-Hernández, D. A., \& Yong, D. 2012, A\&A, 540, A44

Vassiliadis, E., \& Wood, P. R. 1993, ApJ, 413, 641

Watson, C. L. 2006, Society for Astronomical Sciences Annual Symp., 25, 47

Wood, P. R., Bessell, M. S., \& Fox, M. W. 1983, ApJ, 272, 99

Zamora, O., García-Hernández, D. A., Plez, B., \& Manchado, A. 2014, A\&A, 564, L4 
Appendix A: Complete sample
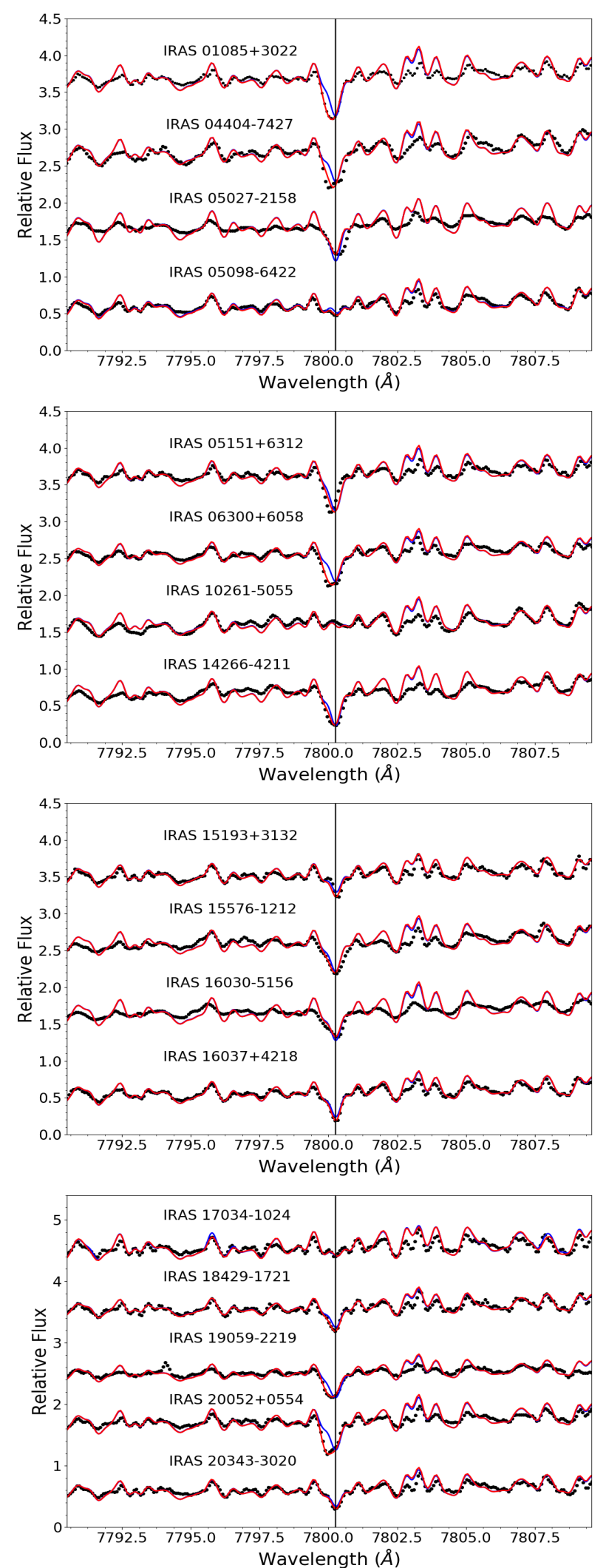
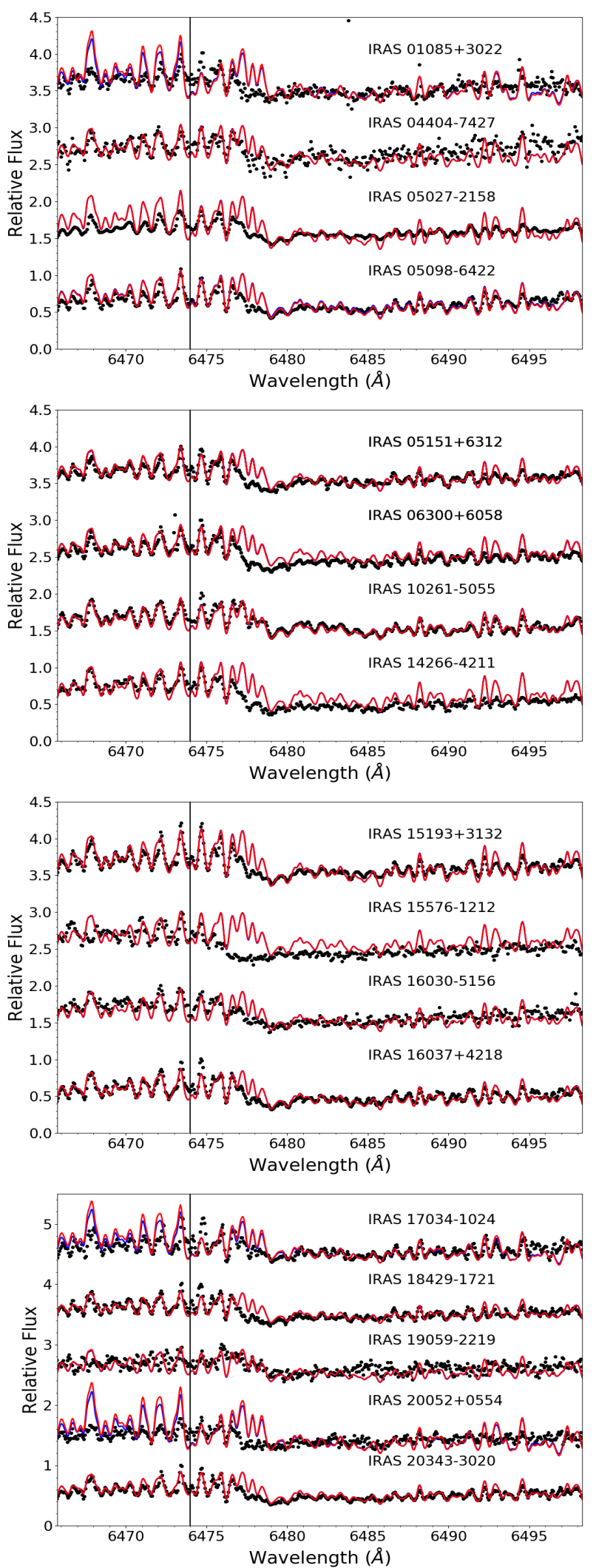

Fig. A.1. Observed spectra (black dots), best hydrostatic (blue lines) and pseudo-dynamical (red lines) fits of our sample of AGB stars in the regions of $7800 \AA \mathrm{Rb}$ I line (left panels) and $6474 \AA \mathrm{ZrO}$ bandhead (right panels). The parameters of the best fit model atmospheres are indicated in Table 3. The plots are displayed in increasing R.A. order. 